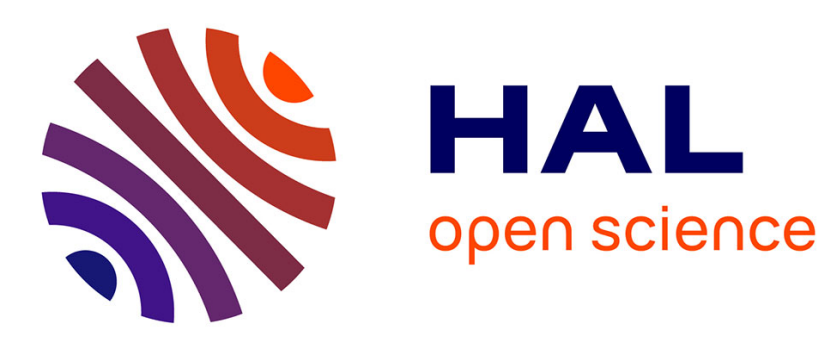

\title{
Triangle-based consistencies for cost function networks
}

Christian Bessiere, Simon de Givry, Thomas Schiex, Thi Hông Hiêp Nguyên

\section{To cite this version:}

Christian Bessiere, Simon de Givry, Thomas Schiex, Thi Hông Hiêp Nguyên. Triangle-based consistencies for cost function networks . Constraints, 2017, 22 (2), pp.230-264. 10.1007/s10601-016-9250-1. lirmm-01374514

\section{HAL Id: lirmm-01374514 https://hal-lirmm.ccsd.cnrs.fr/lirmm-01374514}

Submitted on 30 Sep 2016

HAL is a multi-disciplinary open access archive for the deposit and dissemination of scientific research documents, whether they are published or not. The documents may come from teaching and research institutions in France or abroad, or from public or private research centers.
L'archive ouverte pluridisciplinaire HAL, est destinée au dépôt et à la diffusion de documents scientifiques de niveau recherche, publiés ou non, émanant des établissements d'enseignement et de recherche français ou étrangers, des laboratoires publics ou privés. 


\title{
Triangle-based Consistencies for Cost Function Networks
}

\author{
Hiep Nguyen • Christian Bessiere • \\ Simon de Givry • Thomas Schiex
}

Received: date / Accepted: date

\begin{abstract}
Cost Function Networks (aka Weighted CSP) allow to model a variety of problems, such as optimization of deterministic and stochastic graphical models including Markov random Fields and Bayesian Networks. Solving cost function networks is thus an important problem for deterministic and probabilistic reasoning. This paper focuses on local consistencies which define essential tools to simplify Cost Function Networks, and provide lower bounds on their optimal solution cost. To strengthen arc consistency bounds, we follow the idea of triangle-based domain consistencies for hard constraint networks (path inverse consistency, restricted or max-restricted path consistencies), describe their systematic extension to cost function networks, study their relative strengths, define enforcing algorithms, and experiment with them on a large set of benchmark problems. On some of these problems, our improved lower bounds seem necessary to solve them.
\end{abstract}

Keywords Cost function networks · Weighted CSP · Constraint optimization problems · High order consistencies · Restricted path consistency · Path inverse consistency · Max-restricted path consistency

Hiep Nguyen

MIAT, UR 875, Université de Toulouse, INRA, Castanet-Tolosan, France

E-mail: ifi.nthhiep@gmail.com

Christian Bessiere

University of Montpellier, Montpellier, France

E-mail: bessiere@lirmm.fr

Simon de Givry

MIAT, UR 875, Université de Toulouse, INRA, Castanet-Tolosan, France

E-mail: degivry@toulouse.inra.fr

Thomas Schiex

MIAT, UR 875, Université de Toulouse, INRA, Castanet-Tolosan, France

E-mail: tschiex@toulouse.inra.fr 


\section{Introduction}

Graphical model processing is a central problem in AI. The Cost Function Network framework (CFN [25] as an instance of the valued CSP framework.), where the goal is to optimize the combined cost of local cost functions, captures problems such as weighted MaxSAT, Weighted CSP or Maximum Probability Explanation in probabilistic networks. ${ }^{1}$ CFNs have applications in resource allocation [4], combinatorial auctions, bioinformatics [27,26]..

Dynamic programming approaches such as bucket or cluster tree elimination can be used to tackle such problems but are inherently limited by their exponential time and space behavior on graphical models with high tree-width. Instead, Depth First Branch and Bound allows to keep a polynomial space complexity but requires good (strong and cheap) lower bounds on the minimum cost to be efficient. In the last years, increasingly better lower bounds have been designed by enforcing soft local consistencies on CFNs. Arc consistencies such as $\mathrm{AC}^{*}, \mathrm{DAC}^{*}, \mathrm{FDAC}^{*}, \mathrm{EDAC}^{*}[17]$ or VAC [6] are inspired from arc consistency in hard constraint networks. They have a small order polynomial enforcing time but do not always provide tight enough lower-bounds. The linear programming based OSAC consistency [9] provenly gives the strongest lower bound that can be obtained by arc consistency but is usually too expensive to compute. It now becomes useful to look beyond arc consistencies. Up to now, few higher order consistencies have been proposed for CFNs $[8,12]$.

In this paper, we show that strong soft consistencies can be defined for CFNs by extending hard high order consistencies defined for CSPs. Among hard high order consistencies, the family of triangle-based consistencies (Restricted Path Consistency or RPC, Path Inverse Consistency or PIC, and maxRestricted Path Consistency or maxRPC) are specifically interesting because they have a stronger pruning power than arc consistency, and a cheaper computational cost than other high order consistencies. Their extension to CFNs is however non trivial, and enforcing algorithms create ternary cost functions.

The rest of the paper is organized as follows. Section 2 is a background section on constraint and cost function networks, associated local consistencies and enforcing operations. The next sections focus on our contributions. Section 3 gives a definition of new local consistencies. Section 4 introduces different ways to compare the strength of soft consistencies in general. This is then used to compare the proposed consistencies to each other and to existing consistencies. Section 5 focuses on the algorithms for enforcing the proposed consistencies. The last section gives experimental results when these consistencies are used as pre-processing or maintained during search on a large set of benchmarks. We observe that the strengthened bound provided by triangle consistencies (TRICs) are necessary to solve some problems that could not be solved otherwise.

\footnotetext{
1 We use the terminology of Cost Function Networks by similarity to Constraint Networks. The Weighted Constraint Satisfaction Problem (WCSP) is the problem of solving a CFN. For outsiders, guessing what a Cost Function Network could be, is also much easier.
} 


\section{Background}

A constraint satisfaction problem (CSP) is a triple $(X, D, C)$ where $X$ is a set of $n$ variables, $D$ is a set of $n$ domains (variable $i \in X$ takes values from $\left.D_{i} \in D\right)$, and $C$ is a set of constraints. Each constraint $c_{S} \in C$ defined over a set $S$ of variables specifies the allowed assignments $\tau_{S}$ of values for variables in $S$, denoted by $\tau_{S} \in c_{S} . S$ and $|S|$ are the scope and the arity of the constraint $c_{S}$. For simplicity, $c_{\{i\}}, c_{\{i, j\}}$ are denoted as $c_{i}, c_{i j}$. The constraints $c_{i}, c_{i j}, c_{S}$ with $|S|>2$ are respectively called unary, binary and non-binary. A value $a$ for variable $i$ is denoted by $(i, a)$ or by $i_{a}$. Given a set of variables $S, \ell(S)$ denotes the set of assignments (tuples) of values for variables in $S$, that is, $\ell(S)=D^{S}=\prod_{i \in S} D_{i}$. Given a tuple $\tau_{S}$, a variable $i \in S$ and a subset $S^{\prime} \in S$, $\tau_{S}[i]$ and $\tau_{S}\left[S^{\prime}\right]$ denote the projection of tuple $\tau_{S}$ on $i$ and $S^{\prime}$ respectively. A tuple $\tau_{S}$ is consistent if it satisfies all the constraints whose scope is included in $S$. A solution is a consistent complete assignment. The problem is consistent if it has at least one solution.

Definition 1 (Local consistencies) Given a CSP $P=(X, D, C)$,

- $P$ is arc consistent (AC) if $\forall i \in X, \forall a \in D_{i}, \forall c_{S} \in C$ such that $i \in S$, there exists a tuple $\tau \in \ell(S)$ such that $\tau[i]=a$ and $\tau \in c_{S}$. Such a tuple $\tau$ is called the support of value $(i, a)$ in the constraint $c_{S}$.

- $P$ is restricted path consistent (RPC, [3]) iff it is $\mathrm{AC}$ and $\forall i \in X, \forall a \in D_{i}$, $\forall c_{i j} \in C$ on which $a$ has only one support $b \in D_{j}, \forall k$ linked to $i$ and $j$ by $c_{i k}, c_{j k}$, there exists a value $c \in D_{k}$ such that $(a, c) \in c_{i k}$ and $(b, c) \in c_{j k}$.

- $P$ is path inverse consistent (PIC, [14]) iff it is AC and $\forall i \in X, \forall a \in D_{i}, \forall j, k$ such that $i, j, k$ are linked one-by-one by binary constraints, there exists a value $b \in D_{j}, c \in D_{k}$ such that $(a, b) \in c_{i j},(a, c) \in c_{i k}$ and $(b, c) \in c_{j k}$.

- $P$ is max-restricted path consistent (maxRPC, [11]) iff it is AC and $\forall i \in X$, $\forall a \in D_{i}, \forall c_{i j} \in C, a$ has a support $b \in D_{j}$ such that $\forall k$ linked to both $i, j$ by $c_{i k}, c_{j k}$, there exists a value $c \in D_{k}$ such that $(a, c) \in c_{i k}$ and $(b, c) \in c_{j k}$.

- Let $\Phi$ be a hard consistency and $P$ a CSP. The $\Phi$-closure of $P$ is the CSP $\Phi(P)=\left(X, D_{\Phi}, C\right)$ such that $D_{\Phi}^{X} \subseteq D^{X}, \Phi(P)$ is $\Phi$-consistent, and there does not exist $D^{\prime}$ such that $D_{\Phi}^{X} \subset D^{\prime X}$ and $\left(X, D^{\prime}, C\right)$ is $\Phi$-consistent.

CFNs extend CSPs by associating costs to tuples [25,24]. A CFN is a tuple $(X, D, C, m)$ where $X$ and $D$ are respectively sets of variables and domains, as in classical CSPs. $C$ is a set of cost functions. Each cost function $c_{S} \in C$ assigns non negative integer costs to tuples $\tau_{S} \in \ell(S)$ i.e. $c_{S}: \ell(S) \rightarrow[0 . . m]$ where $m \in\{1, \ldots,+\infty\}$. The addition and subtraction of costs are bounded operations, defined as $a+{ }_{m} b=\min (a+b, m), a-{ }_{m} b=a-b$ if $a<m$ and $m$ otherwise. The combined cost of a tuple $\tau_{S}$ in a CFN $P$ is the sum of costs $\operatorname{Val}_{P}\left(\tau_{S}\right)=\sum_{\left(S^{\prime} \subseteq S\right) \wedge\left(c_{S^{\prime}} \in C\right)} c_{S^{\prime}}\left(\tau_{S}\left[S^{\prime}\right]\right)$, where summation is done using $+_{m} \cdot \tau_{S}$ is inconsistent if $\operatorname{Val}_{P}\left(\tau_{S}\right)=m$, and consistent otherwise. A solution of $P$ is a complete consistent tuple $\tau_{X}$. An optimal solution has minimum $\operatorname{Val}_{P}\left(\tau_{X}\right)$.

It is important to note that CSPs are just CFNs using $m=1$. In such problems, a tuple which receives a cost of 1 is forbidden. A cost of 0 is used for allowed tuples. 
We assume the existence of a unary cost function $c_{i}$ for every variable $i$, and a nullary cost function, noted $c_{\varnothing}$. This constant non-negative cost defines a lower bound on the cost of every solution. A CFN $P$ can be transformed into an equivalent $\mathrm{CFN} P^{\prime}$ (i.e., $\operatorname{Val}_{P}(\tau)=\operatorname{Val}_{P^{\prime}}(\tau) \forall \tau$ ) by applying so-called equivalence-preserving transformations (EPTs) that shift costs between cost functions. The EPT Shift $\left(\tau_{S}, c_{S^{\prime}}, \alpha\right)$ (Algorithm 1) moves an amount of cost $\alpha$ between a cost function $c_{S^{\prime}}$ and a tuple $\tau_{S}$ such that $S \subset S^{\prime}$. The conditions (2) and (3) guarantee that the operation will not create any negative cost in the problem. Shift allows to define the three usual EPTs [10] Project (from $c_{S^{\prime}}$ to $\tau_{S}, \alpha>0$ ), Extend (from $\tau_{S}$ to $c_{S^{\prime}}, \alpha<0$ ) and UnaryProject (from $i$ to $\left.c_{\varnothing}, \alpha>0, S^{\prime}=\{i\}, S=\varnothing\right)$.

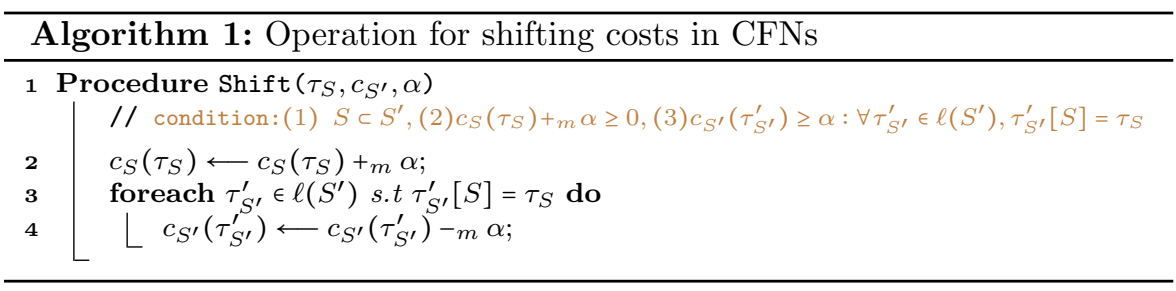

By applying EPTs to an original CFN, it is possible to transform it in an equivalent $\mathrm{CFN}$ that satisfies a given local consistency property. This may increase the lower bound $c_{\varnothing}$. The simplest local consistency, node consistency (NC [16]), requires that $\forall i \in X, \forall a \in D_{i} c_{i}(a)+c_{\varnothing}<m$ and there exists a value $a \in D_{i}$ such that $c_{i}(a)=0$.

Definition 2 (Soft arc consistencies) Given a binary CFN $P=(X, D, C, m)$ and an order $<$ on variables,

- $P$ is arc consistent (AC [24]) iff $\forall i \in X, \forall a \in D_{i}$ and $\forall c_{i j} \in C$, there exists $b \in D_{j}$ such that $c_{i j}(a, b)=0 . b$ is called a (simple) support for $(i, a)$ in $c_{i j} .{ }^{2}$

$-P$ is directional arc consistent (DAC [7]) w.r.t $<$ iff $\forall i, \forall a \in D_{i}, \forall c_{i j}$ such that $i<j$, there exists a value $b \in D_{j}$ such that $c_{i j}(a, b)+c_{j}(b)=0 . b$ is called a full support for $(i, a)$ in $c_{i j}$.

$-P$ is full directional arc consistent (FDAC [19]) w.r.t $<$ iff it is AC and DAC.

- $P$ is existential arc consistent (EAC [17]) iff $\forall i \in X$, there exists a value $a \in D_{i}$ such that $c_{i}(a)=0$ and $\forall c_{i j} \in C$, there exists $b \in D_{j}$ such that $c_{i j}(a, b)+c_{j}(b)=0$. Value $a$ is called the existential arc consistent support of $i$.

$-P$ is existential directional arc consistent (EDAC [17]) iff it is EAC and FDAC.

2 There exists tiny variations on the definition of $\mathrm{AC}$ for CFNs. This paper uses the definition in [20] which simplifies the definition in [10] by not considering the propagation of inconsistent tuples. 
- $\operatorname{Bool}(P)$ is a CSP defined as a $\operatorname{CFN}(X, D, \bar{C}, 1)$ such that $\exists \bar{c}_{S} \in \bar{C}$ iff $\exists c_{S} \in C, S \neq \varnothing$ and $\tau \in \bar{c}_{S} \Leftrightarrow c_{S}(\tau)>0 . P$ is virtual arc consistent (VAC [6]) iff the $\mathrm{AC}-$ closure of $\operatorname{Bool}(P)$ is non-empty.

For simplicity, we restrict ourselves to binary CFNs. A binary CFN is $\mathrm{AC}^{*}$, $\mathrm{DAC}^{*}, \mathrm{FDAC}^{*}, \mathrm{EAC}^{*}, \mathrm{EDAC}^{*}$ if it is $\mathrm{NC}$ and respectively AC, DAC, FDAC, EAC, EDAC [16]. Definitions of soft arc consistencies for non-binary CFNs have been given in $[10,5,21,22]$.

\section{Soft triangle-based consistencies (TRICs)}

In this section, we extend the hard local consistencies RPC, PIC and maxRPC, defined on triangles of variables to CFNs. For each hard consistency, we define six soft variants, also called softening levels: simple, directional, full directional, existential, existential directional, and virtual. This gives rise to eighteen new soft local consistencies. In addition to soft ACs, all these soft versions guarantee the extensibility of arc supports on extra third variables on a so-called witness.

Definition 3 (Witness) Given a value $(i, a)$, a pair of values $\left(i_{a}, j_{b}\right)$ and a variable $k$ linked both to $i$ and $j$,

- A simple witness of $\left(i_{a}, j_{b}\right)$ on $k$ is a value $c \in D_{k}$ such that $c_{i k}(a, c)+$ $c_{j k}(b, c)+c_{i j k}(a, b, c)=0$.

- A full witness of $\left(i_{a}, j_{b}\right)$ on $k$ is a value $c \in D_{k}$ such that $c_{k}(c)+c_{i k}(a, c)+$ $c_{j k}(b, c)+c_{i j k}(a, b, c)=0$.

Definition 4 (Extensibility of a pair of values on a variable) Given a pair of values $\left(i_{a}, j_{b}\right)$ and a variable $k$ linked both to $i$ and $j$,

- $\left(i_{a}, j_{b}\right)$ is simply extensible on $k$ if there exists a simple witness on $k$ for it.

- $\left(i_{a}, j_{b}\right)$ is fully extensible on $k$ if there exists a full witness on $k$ for it.

Definition 5 (Extensibility of a value on a triangle) A triangle is a triple of variables $(i, j, k)$ that are linked one-by-one by binary cost functions. It is noted as $\Delta_{i j k}$. Given a value $(i, a)$ and a triangle $\Delta_{i j k}$.

- $(i, a)$ is simply extensible on triangle $\Delta_{i j k}$ if there exists a simple arc support for $(i, a)$ in $c_{i j}$ that is simply extensible on $k$.

- $(i, a)$ is fully extensible on triangle $\Delta_{i j k}$ if there exists a full arc support for $(i, a)$ in $c_{i j}$ that is fully extensible on $k$.

Definition 6 (Extensibility of a pair of values) For a pair of values $\left(i_{a}, j_{b}\right)$ and an order $<$ on the variables, $\left(i_{a}, j_{b}\right)$ is:

- simply extensible if it is simply extensible on every $k$ linked to both $i$ and $j$.

- fully extensible if it is fully extensible on every $k$ linked to both $i$ and $j$.

- directionally-fully extensible if it is fully extensible on every $k>i$ linked to both $i$ and $j$.

- semi-fully extensible if it is simply extensible on every $k<i$ linked both to $i$ and $j$ and is fully extensible on every $k>i$ linked both to $i$ and $j$. 
Notice that full extensibility implies semi-full extensibility. Semi-full extensibility implies directional-full and simple extensibility. Conversely, both directional-full and simple extensibility do not imply any other extensibility. Examples in Figure 1 illustrate the different extensibilities of pairs of values.

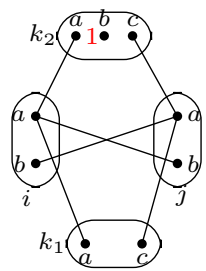

(a)

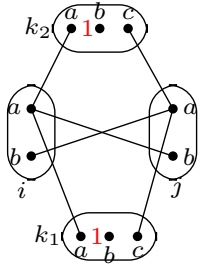

(b)

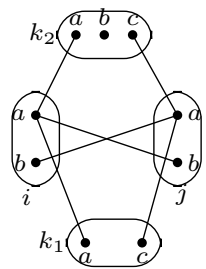

(c)

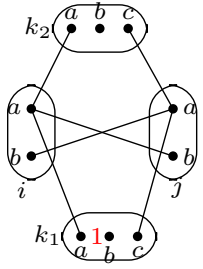

(d)

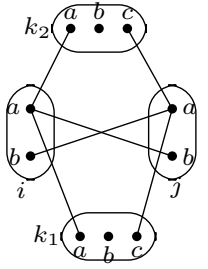

(e)

Fig. 1 Example of different extensibilities of the pair of values $\left(i_{a}, j_{a}\right) . k_{1}<i<j<k_{2}$ An edge appears between pairs of values with a non zero cost. In CFN(a), $\left(i_{a}, j_{a}\right)$ is not simply extensible on $k_{1}$. In CFN (b), $\left(i_{a}, j_{a}\right)$ is simply extensible (on both $k_{1}, k_{2}$ ) but is not directionally-fully extensible (because it is not fully extensible on $\left.k_{2}\right)$. In CFN(c), $\left(i_{a}, j_{a}\right)$ is directionally-fully extensible w.r.t $k_{2}$ but is not semi-fully extensible (because it is not simply extensible on $\left.k_{1}\right)$. In $\mathrm{CFN}(\mathrm{d}),\left(i_{a}, j_{a}\right)$ is semi-fully extensible (fully extensible on $k_{2}$ and simply extensible on $k_{1}$ ) but is not fully extensible (because it is not fully extensible on $k_{1}$ ). In $\operatorname{CFN}(\mathrm{e}),\left(i_{a}, j_{a}\right)$ is fully extensible (on both $k_{1}, k_{2}$ ).

\subsection{Soft restricted path consistencies}

The idea of soft RPC consistencies is to only check the extensibility of pairs of values $\left(i_{a}, j_{b}\right)$ that will make a value soft arc inconsistent if their binary cost becomes positive. If a value $(i, a)$ has only one simple support $(j, b)$ on $c_{i j}$ and this support $\left(i_{a}, j_{b}\right)$ is not extensible on some third variable $k$, every 3 -values tuple over $\{i, j, k\}$, involving $\left(i_{a}, j_{b}\right)$, has a positive combined cost. Because $(j, b)$ is the unique arc support of $(i, a)$, every complete tuple involving $(i, a)$ has a positive cost evaluation. Thus, the unary cost $c_{i}(a)$ can be increased by equivalence preserving transformations.

Definition 7 (Soft restricted path consistencies (Soft RPCs)) Given a CFN $P=(X, C, D, m)$ and an order "<" on variables,

- $P$ is RPC if it is AC and $\forall i \in X, \forall a \in D_{i}, \forall c_{i j} \in C$ on which $(i, a)$ has only one simple arc support $b \in D_{j},\left(i_{a}, j_{b}\right)$ is simply extensible.

- $P$ is directional RPC (DRPC) if it is DAC and $\forall i \in X, \forall a \in D_{i}, \forall c_{i j} \in C$ such that $i<j$ and $(i, a)$ has only one full arc support $b \in D_{j},\left(i_{a}, j_{b}\right)$ is directionally-fully extensible.

- $P$ is full directional RPC (FDRPC) if it is FDAC and $\forall i \in X, \forall a \in D_{i}, \forall c_{i j} \in$ $C$ such that (1) if $i>j$ and $(i, a)$ has only one simple arc support $b \in D_{j}$ then $\left(i_{a}, j_{b}\right)$ is simply extensible, or $(2)$ if $i<j$ and $(i, a)$ has only one full arc support $b \in D_{j}$ then $\left(i_{a}, j_{b}\right)$ is semi-fully extensible. 
- $P$ is existential RPC (ERPC) if $\forall i \in X$, there exists a value $a \in D_{i}$ such that $(1) c_{i}(a)=0,(2) i_{a}$ has a full arc support in every cost function (i.e., $P$ is EAC), and (3) $\forall c_{i j} \in C$ on which $(i, a)$ has only one full arc support $b \in D_{j},\left(i_{a}, j_{b}\right)$ is fully extensible. Such a value $(i, a)$ is the ERPC support for $i$.

$-P$ is existential directional RPC (EDRPC) if it is ERPC and FDRPC.

$-P$ is virtual RPC (VRPC) if the RPC-closure of $\operatorname{Bool}(P)$ is non-empty.

VRPC is defined based on the hard CSP Bool $(P)$ and hard RPC. The other softening levels of RPC differ from each other by (1) the strength of supports (simple or full) (2) the strength of witnesses (simple, full, directional-full, semifull) and (3) the scope of application of these properties (every domain value or one value per domain, every cost function or some specific cost functions).

Example 1 Consider the CFNs in Figure 1.

- CFN(a) is VRPC because the RPC-closure of $\operatorname{Bool}(P)$ is not empty, containing values $\left(i_{b}\right),(j, b),\left(k_{1}, a\right),\left(k_{1}, c\right),\left(k_{2}, a\right),\left(k_{2}, c\right)$. However, it is not RPC because the unique support $\left(i_{a}, j_{a}\right)$ of $(i, a)$ on $c_{i j}$ is not simply extensible on $k_{1}$

- CFN(b) is RPC: both $\left(i_{a}, j_{a}\right)$ and $\left(i_{b}, j_{b}\right)$ (respectively the unique simple arc support of $(i, a),(j, a)$ on $c_{i j}$ and of $(i, b),(j, b)$ on $\left.c_{i j}\right)$ are simply extensible on $k_{1}$ and $k_{2}$ at simple witnesses $\left(k_{1}, b\right)$ and $\left(k_{2}, b\right)$ respectively. However, it is not DRPC because the unique full arc support $\left(i_{a}, j_{a}\right)$ of $(i, a)$ in $c_{i j}$ is not fully extensible on $k_{2}>i$.

- CFN(c) is DRPC because both $\left(i_{a}, j_{a}\right)$ and $\left(i_{b}, j_{b}\right)$ (respectively the unique full arc support of $(i, a)$ in $c_{i j}$ and of $(i, b)$ in $\left.c_{i j}\right)$ are fully extensible on $k_{2}>i$ at $\left(k_{2}, b\right)$. Variable $k_{1}<i$ is not involved in DRPC for $i$. However, it is not FDRPC because the unique full support $\left(i_{a}, j_{a}\right)$ of value $(i, a)$ on $c_{i j}$ is not simply extensible on $k_{1}$.

- CFN(d) is FDRPC where the supports $\left(i_{a}, j_{a}\right)$ and $\left(i_{b}, j_{b}\right)$ are fully extensible on $k_{2}$ at $\left(k_{2}, b\right)$ and simply extensible on $k_{1}$ at $\left(k_{1}, b\right)$. At the same time, it is ERPC where $(i, b),(j, b),\left(k_{1}, a\right),\left(k_{2}, a\right)$ are ERPC supports for variables $i, j, k_{1}$ and $k_{2}$.

\subsection{Soft path inverse consistencies}

We now consider soft path inverse consistencies. They guarantee the extensibility of domain values on triangles of variables. For all triangles $\Delta_{i j k}$ sharing two variables $i, j$ of a cost function $c_{i j}$, PICs require that one of the arc supports of $(i, a)$ in $c_{i j}$ is extensible on $k$. The arc supports of $(i, a)$ that are extensible on different $k$ can be different.

Definition 8 (Soft path inverse consistencies (Soft PICs)) Given a CFN $P=(X, C, D, m)$ and an order $<$ on variables,

$-P$ is PIC if it is AC and $\forall i \in X, \forall a \in D_{i}, \forall \Delta_{i j k},(i, a)$ is simply extensible on $\Delta_{i j k}$. 
- $P$ is directional PIC (DPIC) if it is DAC and $\forall i \in X, \forall a \in D_{i}, \forall \Delta_{i j k}$ such that $i<j, i<k,(i, a)$ is fully extensible on $\Delta_{i j k}$.

- $P$ is full directional PIC (FDPIC) if it is FDAC and $\forall i \in X, \forall a \in D_{i}, \forall \Delta_{i j k}$, $(i, a)$ is fully extensible on $\Delta_{i j k}$ if $i<j, i<k$ and simply extensible on $\Delta_{i j k}$ otherwise.

- $P$ is existential PIC (EPIC) if $\forall i \in X$, there exists a value $a \in D_{i}$ such that (1) $c_{i}(a)=0,(2) i_{a}$ has a full arc support in every cost function (i.e., $P$ is EAC) and $(3)(i, a)$ is fully extensible on every triangle.

$-P$ is existential directional PIC (EDPIC) if it is EPIC and FDPIC.

$-P$ is virtual PIC (VPIC) if the PIC-closure of $\operatorname{Bool}(P)$ is non-empty.

See examples in Figure 2. As in the case of RPC, VPIC is defined based on the hard CSP Bool $(P)$ and hard PIC. The other softening levels differ from each other by the strength of supports, the strength of witnesses, and the scope of application of these properties.

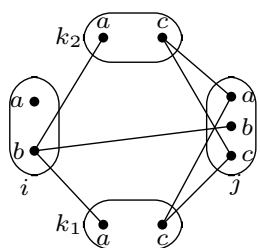

(a) not PIC

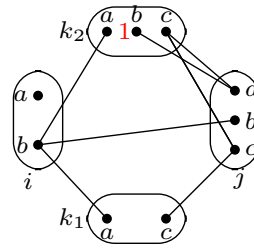

(b) PIC

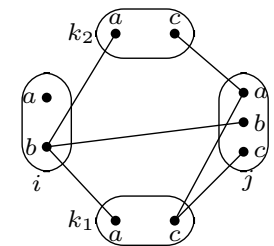

(c) DPIC

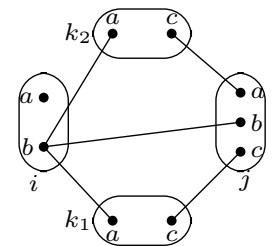

(d) EDPIC

Fig. 2 Example of soft PIC consistencies. $k_{1}<i<k_{2}<j$ and $\exists \Delta_{i j k_{1}}, \Delta_{i j k_{2}}$. The CFN(a) is not PIC because value $(i, b)$ is not simply extensible to triangle $\Delta_{i j k_{1}}$. The $\mathrm{CFN}(\mathrm{b})$ is PIC but is not DPIC because value $(i, b)$ is not fully extensible to triangle $\Delta_{i j k_{2}}$ with $i<j, i<k_{2}$. The CFN(c) is DPIC (because every value in $D_{i}$ can be fully extended to $\Delta_{i j k_{2}}$ (the only triangle concerned by DPIC for $i$ ) but it is not FDPIC (because value $(i, b)$ is not simply extensible to triangle $\left.\Delta_{i j k_{1}}\right)$. The CFN(d) is FDPIC where every variable is simply extensible to 2 triangles and $i$ is fully extensible to $\left(i, j, k_{2}\right)$. The CFN(d) is also EPIC where $(i, a),(j, a),\left(k_{1}, a\right),\left(k_{2}, a\right)$ are respectively EPIC supports of $i, j, k_{1}, k_{2}$.

\subsection{Soft max-restricted path consistencies}

Stronger than PICs, soft max-restricted path consistencies (soft maxRPCs) check the existence of an extensible arc support for each value on each binary cost function whatever the number of arc supports the value has. In contrast to soft PICs, maxRPCs require the extensibility of the same arc support at the same time on all third variables. If value $(i, a)$ has no such extensible arc support in some binary cost function $c_{i j}$, each support $\left(i_{a}, j_{b}\right)$ of $(i, a)$ in $c_{i j}$ is not extensible in some extra variable $k$, i.e. the combined cost of every tuple $\left(i_{a}, j_{b}, k_{c}\right)$ is positive. Thus, the binary cost of every arc support of $(i, a)$ in $c_{i j}$ can be increased by equivalence preserving transformations and then $(i, a)$ will no longer be arc consistent. 
Definition 9 (Soft max-restricted path consistencies (Soft maxR-

PCs)) Given a CFN $P=(X, D, C, m)$ and an order $<$ on the variables,

- $P$ is maxRPC if it is AC and $\forall i \in X, \forall a \in D_{i}, \forall c_{i j} \in C$ there exists a simple arc support $b \in D_{j}$ such that $\left(i_{a}, j_{b}\right)$ is simply extensible.

- $P$ is directional maxRPC (DmaxRPC) if it is DAC and $\forall i \in X, \forall a \in D_{i}$, $\forall c_{i j} \in C$ such that $i<j$, there exists a full arc support $b \in D_{j}$ such that $\left(i_{a}, j_{b}\right)$ is directionally-fully extensible.

- $P$ is full directional maxRPC (FDmaxRPC) if it is FDAC and for $\forall i \epsilon$ $D, \forall a \in D_{i}, \forall c_{i j} \in C(1)$ if $i>j$, there exists a simple arc support $b \in D_{j}$ such that $\left(i_{a}, j_{b}\right)$ is simply extensible. (2) otherwise, if $i<j$, there exists a full arc support $b \in D_{j}$ such that $\left(i_{a}, j_{b}\right)$ is semi-fully extensible.

- $P$ is existential maxRPC (EmaxRPC) if $\forall i \in X$, there exists a value $a \in D_{i}$ such that $(1) c_{i}(a)=0,(2) i_{a}$ has a full arc support in every cost function (i.e., $P$ is EAC) and (3) $\forall c_{i j} \in C$, there exists a full arc support $b \in D_{j}$ such that $\left(i_{a}, j_{b}\right)$ is fully extensible.

$-P$ is existential directional maxRPC (EDmaxRPC) if it is EmaxRPC and FDmaxRPC.

$-P$ is virtual maxRPC (VmaxRPC) if the maxRPC-closure of $\operatorname{Bool}(P)$ is non-empty

See examples in Figure 3. Here again, VmaxRPC is defined based on the hard CSP Bool $(P)$ and hard maxRPC. The other softening levels differ from each other by the strength of supports, the strength of witnesses, and the scope of application of these properties.

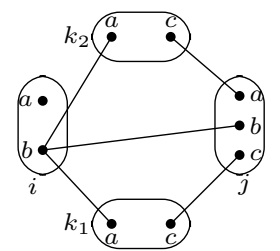

(a) EmaxRPC not maxRPC

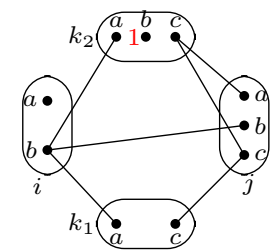

(b) $\operatorname{maxRPC}$ not DmaxRPC

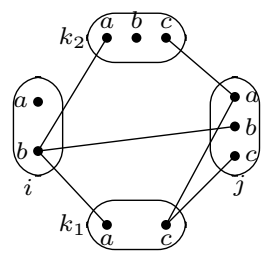

(c) DmaxRPC not FDmaxRPC

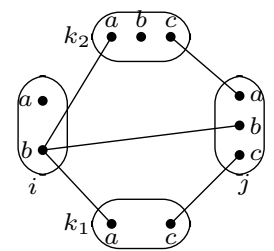

(d) FDmaxRPC EDmaxRPC

Fig. 3 Example of soft maxRPCs. $k_{1}<i<k_{2}<j$ and $\exists \Delta_{i j k_{1}}, \Delta_{i j k_{2}}$. The CFN(a) is not maxRPC because value $(i, b)$ has no arc support in $c_{i j}$ (between $\left(i_{b}, j_{a}\right)$ and $\left(i_{b}, j_{c}\right)$ ) that is simply extensible on both $k_{1}, k_{2}$. The CFN(b) is maxRPC but is not DmaxRPC because value $(i, b)$ has no full arc support in $c_{i j}$ (between $\left(i_{b}, j_{a}\right)$ and $\left(i_{b}, j_{c}\right)$ ) that is fully extensible to $k_{2}$. The CFN(c) is DmaxRPC (because every value in $D_{i}$ has full arc support in $c_{i j}, c_{i k_{2}}$ that is respectively fully extensible on $k_{2}$ and $j$. Triangle $\Delta_{i j k_{1}}$ is not involved in DmaxRPC for $i$ ). The CFN(c) is not FDmaxRPC because value $(i, b)$ has no full support in $c_{i j}$ (between $\left(i_{b}, j_{a}\right)$ and $\left.\left(i_{b}, j_{c}\right)\right)$ that is simply extensible on $k_{1}$. The CFN(d) is both FDmaxRPC and EmaxRPC where $(i, a),\left(k_{1}, a\right),(j, a),\left(k_{2}, a\right)$ are respectively EmaxRPC supports of variables $i, k_{1}, j, k_{2}$. 


\section{Comparing soft local consistencies}

In this section, we compare the strength of the different soft consistencies proposed in the previous section and soft arc consistencies.

Soft consistencies rise specific difficulties when comparison of strength is considered. Most of the consistencies we considered are domain consistencies in the sense that they define properties that values must satisfy and enforcing them may increase unary costs that NC may ultimately use to increase the lower bound $c_{\varnothing}$. However, virtual local consistencies are different because they directly try to increase $c_{\varnothing}$ and do not try to increase unary costs for NC. Thus, the strength of virtual consistencies can be directly measured by the quality of the lower bound provided. For the other soft consistencies, this strength is better measured by the ability to move costs to lower arities. We therefore need to introduce two different order relations between local consistencies to capture this difference between virtual and other consistencies. We denote by $c_{\varnothing}[P]$ the lower bound $c_{\varnothing}$ in a problem $P$.

Furthermore, soft local consistencies are not confluent. A single problem $P$ may have different equivalent problems satisfying a given local consistency property $A$. For a given CFN $P$ and a soft local consistency $A, A(P)$ is therefore defined as the set of problems that can be obtained after enforcing $A$ in $P$. When $P$ already satisfies $A$, we assume that $A(P)=\{P\}$ i.e., that enforcing $A$ on a problem satisfying $A$ does not change $P$ (which is effectively the case for all enforcing algorithms). Similarly, focusing on lower bounds, enforcing a weaker consistency will not change the lower bound.

Definition 10 (Stronger relation) Given two soft consistencies $A$ and $B$,

- $A$ is stronger than $B$, noted by $A \geq B$, iff for every CFN $P$ that satisfies $A, P$ also satisfies $B$, i.e. $B(P)=\{P\}$.

- $A$ is stronger than $B$ in terms of lower bound, noted by $A \geq_{c_{\varnothing}} B$, iff for every CFN $P$ that satisfies $A$ and any $P^{\prime} \in B(P)$, then $c_{\varnothing}\left[P^{\prime}\right]=c_{\varnothing}[P]$.

- $A$ is strictly stronger than $B$, noted $A>B$, iff $A \geq B$ and $\exists$ a CFN $P$ such that $P$ satisfies $B$ and $A(P) \neq\{P\}$.

- $A$ is strictly stronger than $B$ in terms of lower bound, noted $A>_{c_{\varnothing}} B$, iff $A \geq_{c_{\varnothing}} B$ and $\exists$ a CFN $P$ such that $P$ satisfies $B$ and $\forall P^{\prime} \in A(P), c_{\varnothing}\left[P^{\prime}\right]>$ $c_{\varnothing}[P]$.

We first show that $\geq$ entails $\geq_{c_{\varnothing}}$.

Proposition 1 Given two soft consistencies $A$ and $B$, if $A \geq B$ then $A \geq_{c_{\varnothing}} B$.

Proof Because $A \geq B, B(P)=\{P\}$ for every $P$ that satisfies $A$. So we have $\forall P^{\prime} \in B(P), c_{\varnothing}\left[P^{\prime}\right] \geq c_{\varnothing}[P]$ and thus $A \geq_{c_{\varnothing}} B$.

Similarly to the stronger and strictly stronger relations for hard consistencies, our relations for soft consistencies are transitive.

Proposition 2 (Transitivity) Given three soft consistencies $A, B$, and $C$, a. If $A \geq B$ and $B \geq C$ then $A \geq C$. 
b. If $A>B$ and $B>C$ then $A>C$.

c. If $A>B$ and $B \geq_{c_{\varnothing}} C$ then $A \geq_{c_{\varnothing}} C$.

Proof a. Let $P$ be a CFN that satisfies $A$. Because $A \geq B$ and $P$ satisfies $A, B(P)=\{P\}$, i.e. $P$ also satisfies $B$. Because $B \geq C$ and $P$ satisfies $B$, $C(P)=\{P\}$. Thus, if $P$ satisfies $A, C(P)=\{P\}$, i.e. $A \geq C$.

b. (1) Because > implies $\geq$, we have $A \geq B$ and $B \geq C$. So $A \geq C$ from the property (a). (2) Because $A>B$, there exists a CFN $P$ satisfying $B$ and $A(P) \neq\{P\}$. Because $P$ satisfies $B$ and $B \geq C, P$ also satisfies $C$. Thus there exists $P$ that satisfies $C$ and $A(P) \neq\{P\}$. So $A>C$.

c. Because $>$ implies $\geq$, we have $A \geq B$. Let $P$ be a CFN that satisfies $A, P$ also satisfies $B$. Because $B \geq_{c_{\varnothing}} C$ and $P$ satisfies $B, \forall P^{\prime} \in C(P), c_{\varnothing}\left[P^{\prime}\right]=$ $c_{\varnothing}[P]$. Thus, for every CFN which satisfies $A, \forall P^{\prime} \in C(P), c_{\varnothing}\left[P^{\prime}\right]=c_{\varnothing}[P]$. i.e. $A \geq_{c_{\varnothing}} C$.

To show that a soft consistency $A$ is not stronger (resp. not stronger in terms of lower bound than $B$ ), it is enough to show that there exists a CFN $P$ in which $A$ holds and $B$ does better than $A: B(P) \neq\{P\}$ (resp. $\exists P^{\prime} \in$ $\left.B(P), c_{\varnothing}\left[P^{\prime}\right] \neq c_{\varnothing}[P]\right)$.

Two consistencies $A$ and $B$ are incomparable iff $A$ is not stronger than $B$ and $B$ is not stronger than $A$.

Definition 11 (Incomparable relation) Given two soft consistencies $A$ and $B$,

- $A$ and $B$ are incomparable, noted $A \nsucc B$, iff $A \nsucceq B$ and $B \nsucceq A$

- $A$ and $B$ are incomparable in terms of lower bound, noted $A \psi_{c_{\varnothing}} B$, if $A \Varangle_{c_{\varnothing}} B$ and $A \Varangle_{\varnothing \varnothing} B$

Figure 4 is the Hasse diagram that summarizes the relations among soft ACs, RPCs, PICs and maxRPCs. A row of the graph corresponds to six soft consistencies associated with a same hard consistency and a column corresponds to the soft consistencies at a same softening level. A directed path from a consistency $A$ to $B$, without or with dashed arrow, respectively means that $A>B$ or $A>c_{\varnothing} B$. If there does not exist any directed path between $A$ and $B$, they are incomparable. First, we consider the relation between virtual consistencies and domain consistencies. Then, domain consistencies are considered according to the rows and the columns of the graph.

Theorem 1 Given two hard local consistencies $\bar{A}, \bar{B} \in\{A C, R P C, P I C, \max R P C\}$, if we denote by $V A, V B$ their corresponding virtual consistencies and $A, B$ any other softening level of $\bar{A}$ and $\bar{B}$,

a. $V A>c_{\varnothing} A$.

b. If $\bar{A}>\bar{B}$ then

b1 $V A>V B$

b2 $V A>_{c_{\varnothing}} B$.

Proof a. We first prove that VA $\geq_{\varnothing} A$ by contradiction. Suppose that there exists a CFN $P$ satisfying VA and enforcing $A$ can still increase $c_{\varnothing}$ from 


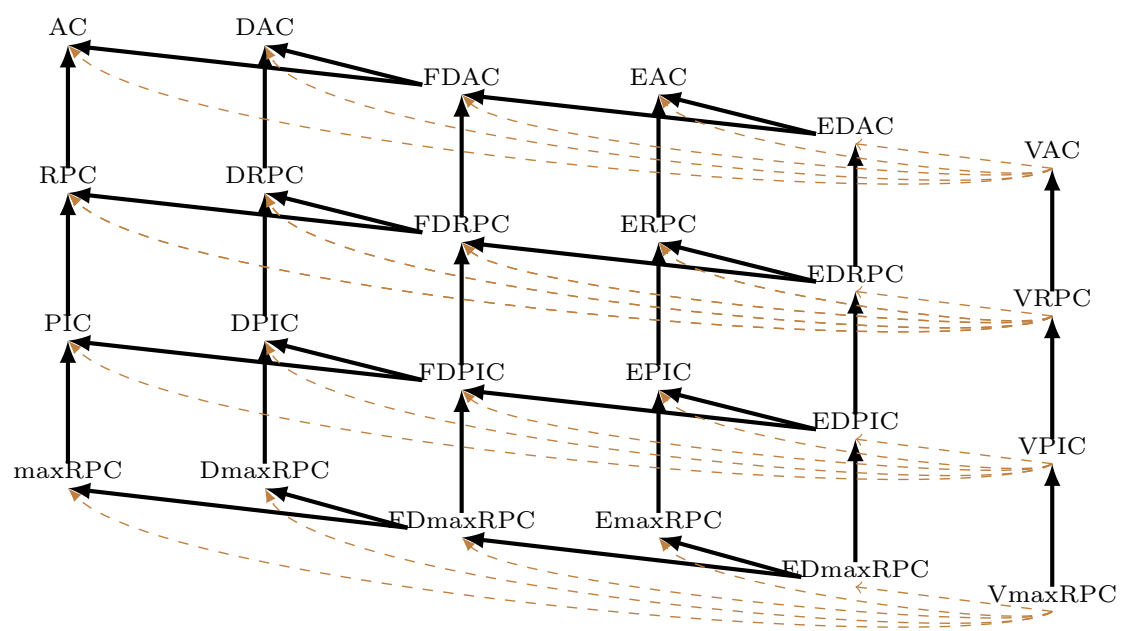

Fig. 4 Hasse diagram of relations between soft consistencies

$$
\begin{array}{ll}
A \rightarrow B: A>B & A \longrightarrow B \rightarrow C \text { implies } A \rightarrow C \\
A \rightarrow B: A>_{C \varnothing} B & A \longrightarrow B \rightarrow C \text { implies } A \rightarrow C
\end{array}
$$

a variable $x_{\varnothing}$. All values and tuples whose costs have been necessary for increasing $c_{\varnothing}$ by $A$ are also forbidden when enforcing $\bar{A}$ in the classic CSP $\operatorname{Bool}(P)$. So, if we eliminate these values and tuples in the same order that costs are moved by $A$ in $P, x_{\varnothing}$ will be wiped-out in $\operatorname{Bool}(P)$. Thus $P$ is not VA and the assumption is false. This means that VA $\geq_{c_{\varnothing}} A$. Secondly, Figure 12 shows a problem that satisfies every non-virtual variant of AC, RPC, PIC, maxRPC but not the virtual ones. Enforcing the virtual one will lead to a strictly stronger $c_{\varnothing}$.

b. Consider first the case of $V B$. First, we prove that VA $\geq V B$. Let $P$ be a CFN which is VA. The $\bar{A}$-closure of $\operatorname{Bool}(P)$ is not empty. Because $\bar{A} \geq \bar{B}$, the $\bar{B}$-closure of $\operatorname{Bool}(P)$ will be not empty. Thus, $P$ also satisfies VB, i.e. $\operatorname{VB}(P)=\{P\}$. Now we prove that $\mathrm{VA} \neq V B$, i.e. $\operatorname{VmaxRPC}>\mathrm{VPIC}>$ VRPC > VAC. Figures 5, 6, 7 respectively show a CFN which is VAC but not VRPC, VRPC but not VPIC, VPIC but not VmaxRPC and $c_{\varnothing}$ can be increased by the unsatisfied consistencies.

We now consider the case of any other soft consistency $B$ associated with $\bar{B}$. From VA $>\mathrm{VB}$ (just above) and from $V B>_{c_{\varnothing}} B$ (Theorem 1(a)), by Proposition $2(\mathrm{c})$, we deduce that $\mathrm{VA} \geq_{c_{\varnothing}} B$. Now, we will prove that VA $>_{c_{\varnothing}} B$. Because $V B>_{c_{\varnothing}} B$, there exists a CFN $P$ such that $P$ is $B$ and $V B$ can still increase the lower bound $c_{\varnothing}[P]$. This means that the $\bar{B}$-closure of $\operatorname{Bool}(P)$ is empty. Because $\bar{A}>\bar{B}$, the $\bar{A}$-closure of $\operatorname{Bool}(P)$ is also empty. Thus, enforcing VA on $P$ will increase $c_{\varnothing}$ while $P$ satisfies $B$.

The following theorem shows that given a softening level, the corresponding soft maxRPC is strictly stronger than the corresponding soft PIC, which 
is strictly stronger than the corresponding soft RPC, which itself is strictly stronger than the corresponding soft AC.

\section{Theorem 2 (Vertical comparison)}

a. $\max R P C>P I C>R P C>A C$.

b. $D \max R P C>D P I C>D R P C>D A C$.

c. $F D \max R P C>F D P I C>F D R P C>F D A C$.

d. $E \max R P C>E P I C>E R P C>E A C$.

e. $E D \max R P C>E D P I C>E D R P C>E D A C$.

Proof First, we can note that the "stronger than" relation $\geq$ holds between the considered pairs of consistencies, based on their definition: at each softening level, the soft variant of maxRPC implies the soft variant of PIC. The same applies for PIC and RPC, as well as RPC and AC. Second, we prove the "strictly stronger than" relation between them by showing CFNs in which the weaker consistencies hold while the stronger ones do not.

a. Figure 5 shows a CFN that satisfies AC but does not satisfy RPC. Figure 6 shows a CFN that satisfies RPC but does not satisfy PIC. Figure 7 shows a CFN that satisfies PIC but does not satisfy maxRPC. Thus maxRPC > $\mathrm{PIC}>\mathrm{RPC}>\mathrm{AC}$.

b-e. The proof is similar to that for (a) by using Figure 5, 6 and 7 .

The following theorem will show that for any hard consistency: (1) the associated existential directional consistency is strictly stronger than both the existential and the full directional ones, (2) the associated full directional consistency is strictly stronger than both the non-directional and the directional ones, (3) other pairs of soft consistencies are incomparable.

Theorem 3 (Horizontal comparison) Given two different hard consistencies $\bar{A}$ and $\bar{B}$ in $\{A C, R P C, P I C, \max R P C\}$, given $A, D A, F D A, E A, E D A$ the simple, directional, full directional, existential, existential directional variant of $\bar{A}$ and $B, D B, F D B$ the simple, directional, full directional variant of $\bar{B}$,

a. (column 2-1): $A \Varangle D B$

b. (column 3-1,2): $F D A>A, D A$

c. (column 4-1,2,3): $E A$ * $B, D B, F D B$

d. (column 5-3,4): $E D A>F D A, E A$

Proof a. $A \nsim D B$ : using Figures 8 and 9 .

b. $F D A>A, D A$. The "stronger than" relation $\geq$ is implied by the definition of the consistencies. $F D A>A$ : Figure 8 shows a problem which is maxRPC, PIC, RPC, AC but is not FDmaxRPC, FDPIC, FDRPC, FDAC. FDA > $D A$ : Figure 9 shows a problem which is DmaxRPC, DPIC, DRPC, DAC but is not FDmaxRPC, FDPIC, FDRPC, FDAC.

c. $E A \neq B, D B, F D B$ : using Figures 10 and 11 .

d. $E D A>F D A, E A$. The proof directly follows from the definitions. 
Theorem 4 (Incomparability) For any pair of consistencies which is not covered by the three previous theorems, the consistencies are incomparable.

Proof - FDAC * RPC,PIC, maxRPC, DRPC,DPIC,DmaxRPC: using Figures 5,8 and 9 .

- FDRPC $*$ PIC, maxRPC, DPIC,DmaxRPC: using Figures 6, 8 and 9.

- FDPIC $*$ maxRPC, DmaxRPC: using Figures 7, 8 and 9 .

- EDAC $*(\mathrm{E} / \mathrm{FD} / \mathrm{D} /-)(\mathrm{RPC} / \mathrm{PIC} / \operatorname{maxRPC})$ : using Figures 5, 10 and 11.

- EDRPC $*$ EPIC, EmaxRPC, FDPIC, FDmaxRPC, DPIC, DmaxRPC, PIC, maxRPC: using Figures 6, 10 and 11.

- EDPIC $*$ EmaxRPC, FDmaxRPC, DmaxRPC, maxRPC: using Figures 7, 10 and 11.

$-\mathrm{VAC} \kappa_{c_{\varnothing}}(\mathrm{ED} / \mathrm{E} / \mathrm{FD} / \mathrm{D} /-)(\mathrm{RPC} / \mathrm{PIC} / \mathrm{maxRPC}):$ using Figures 5 and 12.

- VRPC $*_{c_{\varnothing}}(\mathrm{ED} / \mathrm{E} / \mathrm{FD} / \mathrm{D} /-)(\mathrm{PIC} / \mathrm{maxRPC})$ : using Figures 6 and 12.

- VPIC $\kappa_{\varnothing}(\mathrm{ED} / \mathrm{E} / \mathrm{FD} / \mathrm{D} /-) \operatorname{maxRPC}$ : using Figures 7 and 12.

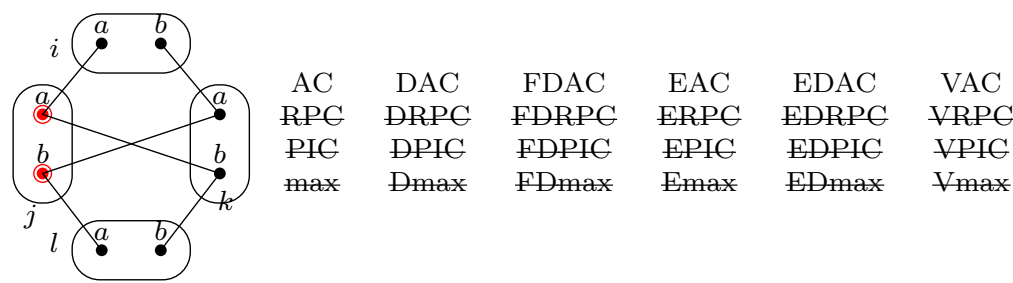

Fig. 5 A CFN that satisfies all arc consistencies but does not satisfy any soft RPC (hence does not satisfy any soft PIC, maxRPC). $j<k<i<l$. The table on the right indicates which consistencies are satisfied or not (strikethrough). maxRPC is briefly written as max, and the same for other variants of maxRPCs. The problem does not satisfy any soft RPC because of variable $j$ (the unique support $\left(j_{a}, k_{a}\right)$ of $(j, a)$ in $c_{j k}$ is not simply extensible on $i$ and the unique support $\left(j_{b}, k_{b}\right)$ of $(j, b)$ is not simply extensible on $\left.l\right)$.

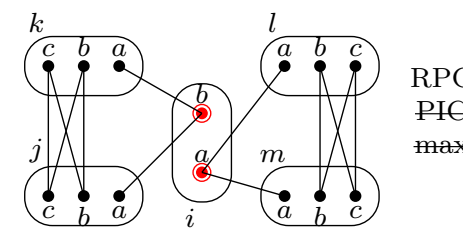

$\begin{array}{llllll}\text { RPC } & \text { DRPC } & \text { FDRPC } & \text { ERPC } & \text { EDRPC } & \text { VRPC } \\ \text { PIC } & \text { DPIC } & \text { FDPIC } & \text { EPIC } & \text { EDPIC } & \text { VPIC } \\ \text { max } & \text { Dmax } & \text { FDmax } & \text { Emax } & \text { EDmax } & \text { Vmax }\end{array}$

Fig. 6 A CFN that satisfies all RPC consistencies but does not satisfy any PIC consistency. $i<j<k<l<m$. Every value of $i$ satisfies RPC consistencies because it has more than 2 full (hence simple) arc supports in $c_{i k}, c_{i j}, c_{i l}, c_{i m}$. The problem does not satisfy any PIC consistency because of variable $i$ (value $(i, a)$ is not simply (hence not fully) extensible to triangle $\Delta_{i l m}$ while $(i, b)$ is not simply (hence not fully) extensible to triangle $\Delta_{i j k}$ ). 


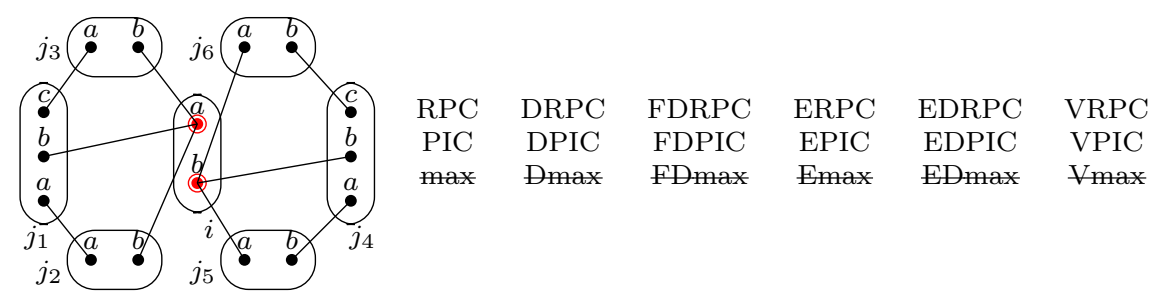

Fig. 7 A CFN that satisfies all PIC consistencies but does not satisfy any maxRPC consistency. $i<j_{1}<j_{2}<j_{3}<j_{4}<j_{5}<j_{6}$. There are only zero unary costs in this problem, thus simple and full supports (or witnesses) are identical. The problem is EDPIC since both $(i, a),(i, b)$ can be fully extended to all 4 triangles. However, the problem does not satisfy any maxRPC consistency because of variable $i$ (no arc support of value $(i, a)$ in $c_{i j_{1}}$ can simultaneously be extended on $\Delta_{i j_{1} j_{2}}$ and $\Delta_{i j_{1} j_{3}}$, the same for value $(i, b)$ in $\left.c_{i j_{4}}\right)$.

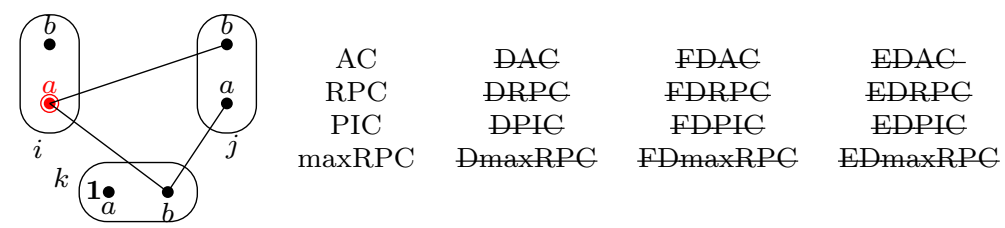

Fig. 8 A CFN which is non-directional consistent but is directional inconsistent for order $i<j<k$. The problem is not DAC because value $(i, a)$ has no full arc support in $c_{i k}$. Therefore, it does not satisfy FDAC, EDAC, FDRPC, EDRPC, FDPIC, EDPIC, FDmaxRPC, EDmaxRPC. However, the problem is maxRPC (hence PIC, RPC) because it is AC and every domain value is simply extensible to the triangle.

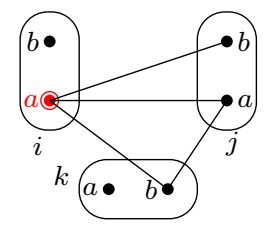

$\begin{array}{cccc}\text { AC } & \text { DAC } & \text { FDAC } & \text { EDAC } \\ \text { RPC } & \text { DRPC } & \text { FDRPC } & \text { EDRPC } \\ \text { PIC } & \text { DPIC } & \text { FDPIC } & \text { EDPIC } \\ \text { maxRPC } & \text { DmaxRPC } & \text { FDmaxRPC } & \text { EDmaxRPC }\end{array}$

Fig. 9 A CFN which is directional consistent $(i>j>k)$ but is non-directional inconsistent. The problem is not $\mathrm{AC}$ because $(i, a)$ has no arc support in $c_{i j}$. However, the problem is DAC because every value of $j$ and $k$ has full arc support in $c_{j i}, c_{k i}$. Moreover, the problem is DmaxRPC (hence DPIC, DRPC) because every value of $j$ and $k$ can be fully extended on the triangle (in the triangle $\Delta_{i j k}$, only the smallest variable $k$ and $c_{k i}, c_{k j}$ are concerned by triangle-based directional consistencies).

\section{Algorithms}

In this section, we present algorithms for enforcing soft PIC, DPIC, FDPIC, EPIC, EDPIC, maxRPC, DmaxRPC, FDmaxRPC, EmaxRPC, and EDmaxRPC. Soft RPCs have not been implemented because they are weaker than their PIC and maxRPC counterparts and because it is costly to maintain the uniqueness of arc supports per value in each cost function -arc supports can be iteratively created and broken when EPTs are applied.

For a value $(i, a)$ that does not satisfy a given TRIC (triangle consistency), the common idea is to create a support for a value $(i, a)$ on $c_{i j}$ that is 


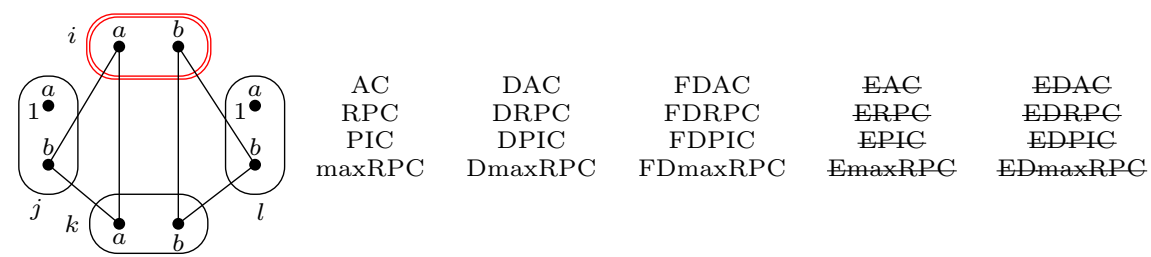

Fig. 10 A CFN which is full directional consistent but is existential inconsistent for $l<$ $j<k<i$. The problem is not EAC (hence not ERPC, EPIC, EmaxRPC) because of value $i\left(i_{a}\right.$ has no full support in $c_{i j}$ while $i_{b}$ has no full support in $c_{i l}$ ). However, the problem is FDmaxRPC (hence FDPIC, FDRPC) because it is FDAC and every value of $i, k$ can be simply extended to both triangles and every value of $j, l$ can be fully extended to $\Delta_{j i k}$ and $\Delta_{l i k}$ respectively.
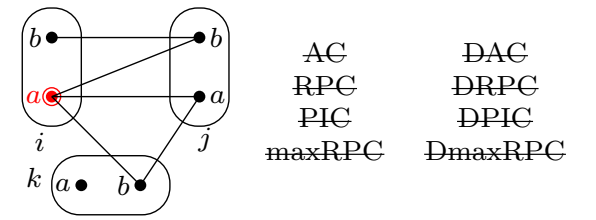

FDAC
FDRPC
FDPIC
FDmaxPC

EAC
ERPC
EPIC

EDAC

EDRPC

maxRPG DmaxRPG FDmaxRPG EmaxRPC EDmaxRPG

Fig. 11 A CFN which is existential consistent but not full directional consistent for $i>j>k$. The problem is not AC (hence is not RPC, PIC, maxRPC) because of value $(i, a)$ (no arc support in $c_{i j}$ ) and is not DAC (hence is not DRPC, DPIC, DmaxRPC) because of value $(j, b)$ (no full arc support in $c_{i j}$ ). However, the problem is EmaxRPC (hence EPIC, ERPC, $\mathrm{EAC})$ where $(i, b),(j, a),(k, a)$ are respectively EmaxRPC supports of $i, j, k$.

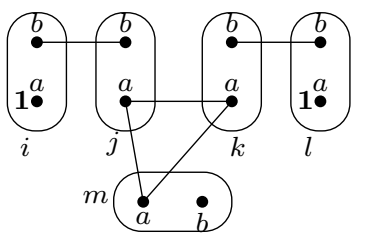

$\begin{array}{cccccc}\text { AC } & \text { DAC } & \text { FDAC } & \text { EAC } & \text { EDAC } & \text { VAC } \\ \text { RPC } & \text { DRPC } & \text { FDRPC } & \text { ERPC } & \text { EDRPC } & \text { VRPC } \\ \text { PIC } & \text { DPIC } & \text { FDPIC } & \text { EPIC } & \text { EDPIC } & \text { VPIC } \\ \text { max } & \text { Dmax } & \text { FDmax } & \text { Emax } & \text { EDmax } & \text { Vmax }\end{array}$

Fig. 12 A CFN which is existential directional but is not virtual consistent $l<i<j<k<m$. The problem is not VAC (hence not VRPC, VPIC, VmaxRPC) because AC makes Bool(P) wiped-out at $j$ or $k$. Conversely, the problem is EDmaxRPC where variables $j, m, l$ are FDmaxRPC in $\Delta_{i j m}$ and $i_{b}, j_{a}, k_{a}, l_{b}, m_{b}$ are EmaxRPC supports of variables.

also extensible on variables $k$ by shifting costs in triangles $\Delta_{i j k}$ (consisting of binary, ternary and possibly unary costs) to $c_{i}$. We denote by $\Delta_{i j k}(a, b, c)=$ $c_{i j}(a, b)+c_{j k}(b, c)+c_{i k}(a, c)+c_{i j k}(a, b, c)$ the combined cost defined by the sum of binary and ternary costs involved in tuple $\left(i_{a}, j_{b}, k_{c}\right)$, where $c_{i j k}(a, b, c)=0$ if $c_{i j k}$ does not exist. Algorithm 2 presents all the basic operations for shifting costs inside triangles and pruning values.

- Extend2To3 $\left(i, a, j, b, c_{i j k}, \alpha\right)$ extends a cost of $\alpha$ from a pair of values $\left(i_{a}, j_{b}\right)$ to a ternary cost function $c_{i j k}$.

- Project3To2 $\left(c_{i j k}, i, a, j, b, \alpha\right)$ projects a cost of $\alpha$ from $c_{i j k}$ on $\left(i_{a}, j_{b}\right)$.

- Project3To1 $\left(c_{i j k}, i, a, \alpha\right)$ projects a cost of $\alpha$ from $c_{i j k}$ on a value $(i, a)$.

- Extend1To2 $\left(i, a, c_{i j}, \alpha\right)$ extends a cost of $\alpha$ from a value $(i, a)$ to $c_{i j}$.

- Project2To1 $\left(c_{i j}, i, a, \alpha\right)$ projects a cost of $\alpha$ from $c_{i j}$ on a value $(i, a)$ 
- PruneVars() removes all inconsistent values having unary cost equal to $m$.

The queues $Q, P, S, T$ store variables or cost functions which had some change in domain or in cost. They will be used for the propagation of changes in our algorithm.

- $Q$ stores variables $i$ such that some value of $D_{i}$ has been deleted (Procedure PruneVars (), line 24).

- $P$ stores variables $i$ such that some value of $D_{i}$ has increased its cost from 0 (Procedure Project3To1 at line 13 and Project2To1 at line 17

- $S$ is an auxiliary queue with the same contents as $P$ (Procedure Project3To1 at line 13 and Procedure Project2To1 at line 17). It is used to efficiently build the propagation queue $R$ which contains variables that need to be checked for the existential consistency. These are all variables of $S$ (those that have values which cost increased from 0 ) and their neighbors because: (1) for $i \in S$, the value in $D_{i}$ that has increased its unary cost may be the existential support of $i$ and (2) the existential support of neighbor variables $j$ may be fully supported by this value.

- $T$ contains binary cost functions $c_{i j}$ that have been modified (because of a unary cost extension in Procedure Extend1To2, line 4) for which $i, j$ and their common neighbors may have lost simple support/witness and need to be revised.

\subsection{Enforcing PICs}

\subsubsection{Enforcing PIC supports}

Simple PIC supports are enforced by Procedure findPICSupport in Algorithm 3. To create a simple PIC support for a value $i_{a}$ on $\Delta_{i j k}$, binary and ternary costs involved in $\Delta_{i j k}$ are moved to $i_{a}$ in such a way that there is a tuple $\left(i_{a}, j_{b}, k_{c}\right)$ whose ternary and binary costs decrease to 0 . The order for moving costs is presented in Figure 13. First, binary costs $c_{i j}, c_{i k}, c_{j k}$ are extended on ternary cost function $c_{i j k}$ by Procedure Extend2To3 (lines 10-12). Then, ternary $\operatorname{costs} c_{i j k}$ are projected on $i_{a}$ by Procedure Project3To1 (line 13). The maximum cost that can be projected on each value $a \in D_{i}$ is stored in $P_{i}[a]$. It is computed based on the available binary and ternary costs (line 3). Binary cost extensions $E_{i j}, E_{i k}, E_{j k}$ are then computed based on $P_{i}[a]$ and the ternary and binary costs (on the two other sides of the triangle, see lines 4-9). The extensions should be sufficiently large so that later projections of $P_{i}[a]$ will not create negative costs and sufficiently small so that a zero triangle cost remains after projection: there should exist values $k_{c}, j_{b}$ and $i_{a}$ such that the final resulting ternary cost $c_{i j k}(a, b, c)+E_{i j}(a, b)+E_{i k}(a, c)+E_{j k}(b, c)-P_{i}[a]=0$. Each computed pairwise extended cost $E \cdot \cdot(\cdot, \cdot)$ is sufficient to satisfy the maximum cost requirements on the third variable. Since these extensions are supposed to be done sequentially, line 7 subtracts $E_{i j}(a, b)$, which will be included in the ternary cost, and does not require $c_{i j}(a, b)$. The same reasoning applies for line 9 , for both previous extensions. 


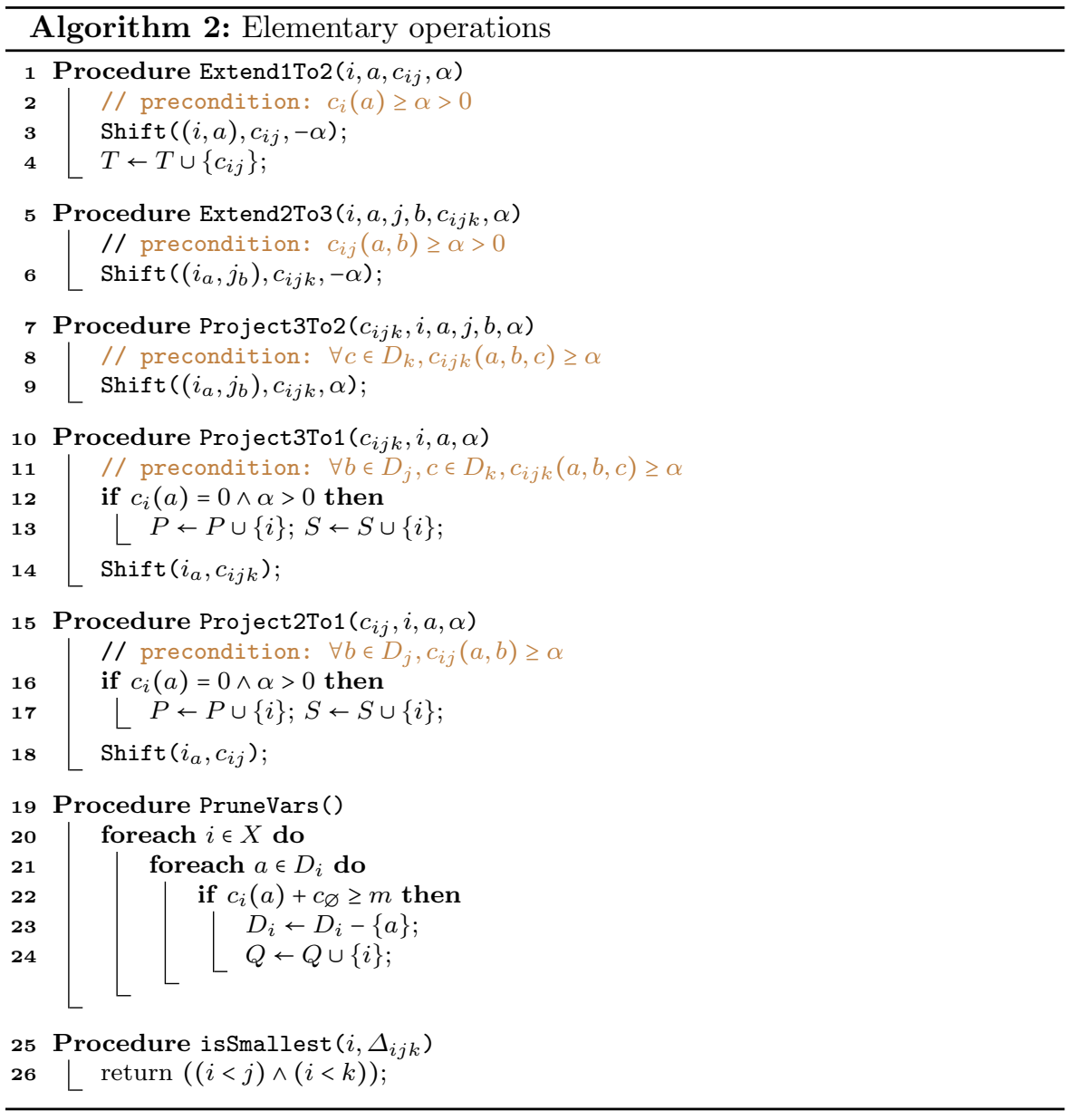

In the end, binary cost extensions on ternary functions do not lead to the loss of ternary AC supports. Moreover, binary cost extensions do not lead to the loss of PIC supports because PIC supports involve only zero binary costs which cannot be used for extension.

Full PIC supports are similarly enforced by Procedure findFullPICSupport in Algorithm 3. The difference is that unary costs on $j, k$ are extended on binary functions $c_{i j}$ and $c_{i k}$ by Procedure Extend1To2, in order to create full PIC supports with zero unary costs (lines 23,24 respectively). Then binary and ternary costs are moved to $i_{a}$ as for simple PIC supports (line 25). The order in which costs are moved to enforce full PIC supports is also visible in Figure 13. The unary costs of $j, k$ are taken into account for the computation of $P_{i}[a]$ as well as for the computation of unary cost extensions $E_{j}, E_{k}$ (lines $18,20,22)$. As for binary extensions, unary cost extensions should be sufficiently large to avoid the creation of negative costs by later projections of $P_{i}[a]$ and 


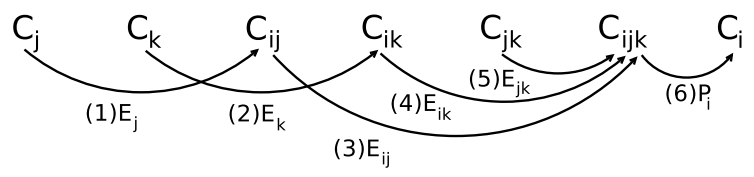

$E_{\mathrm{x}}$ : extension from the cost function of scope $\mathrm{x}$

$P_{x}$ : projection to the cost function of scope $x$

Fig. 13 The order of cost movements for enforcing simple or full PIC supports on variable $i$, where unary cost extensions are not included in the enforcement of simple PIC supports. The arcs indicate the direction of cost movements and the numbers under the arcs indicate the order in which the corresponding cost movements are performed.

small enough so that the the final binary $\operatorname{costs} c_{i j}(a, b)+E_{j}(b)-E_{i j}(a, b)$ and $c_{i k}(a, c)+E_{k}(c)-E_{i k}(a, c)$ are equal to 0.

Therefore, unary cost extensions on binary functions cannot lead to the loss of binary AC supports. However, unary cost extensions on binary functions can lead to the loss of simple PIC supports, thus modified binary functions are stored in the list $T$ in order to later enforce PIC supports for related values.

Example 2 Consider the CFN(a) in Figure 14. It has 4 variables $i<j<k<l$ and 5 binary cost functions $c_{i j}, c_{i k}, c_{i l}, c_{j k}, c_{j l}$. Binary costs are represented by edges (continuous line) and ternary costs are represented by hyper-edges (dashed lines for $c_{i j k}$ and dotted lines for $c_{i j l}$ ). The absence of (hyper)edges indicates a zero cost. The initial problem is FDAC but not FDPIC because value $(i, a)$ is not fully extensible on $\Delta_{i j k}$. Now, consider enforcing full PIC supports for the values of $i$. Procedure findFullPICSupport $(i, j, k)$ computes the amounts of cost for projections/extensions: $P_{i}[a]=E_{j}[b]=1$. Other shifted costs are zero. After extending a cost of 1 from $(j, b)$ on $c_{i j}$, it will call Procedure findPICSupport $(i, j, k)$, compute the amounts of shifted cost as follows:

$$
P_{i}[a]=E_{i j}[a, b]=E_{i k}[a, a]=E_{j k}[a, b]=1 .
$$

and perform the cost shifts. The resulting problem, presented in Sub-figure 14(d) is still not FDPIC because value $(i, b)$ cannot be fully extended on triangle $\Delta_{i j l}$. Then Procedure findFullPICSupport $(i, j, l)$ computes and performs the following cost shifting:

$$
P_{i}[b]=E_{i j}[b, b]=E_{i l}[b, a]=E_{j l}[a, b]=1 .
$$

The final problem, presented in Sub-figure $14(\mathrm{~g})$ is FDPIC. Contrarily to hard PIC, enforcing simple and full PIC supports can create new ternary functions, e.g., $c_{i j k}, c_{i j l}$. Whenever a binary cost need to be extended to a ternary cost function that does not exist, the ternary cost function needs to be created and initialized with an empty cost for every tuple.

\subsubsection{Soft PIC algorithms}

Enforcing EDPIC requires enforcing PIC, DPIC, and EPIC simultaneously. We thus only present an algorithm for EDPIC. PIC, DPIC, FDPIC, and EPIC algorithms can be derived by removing blocks of code. 


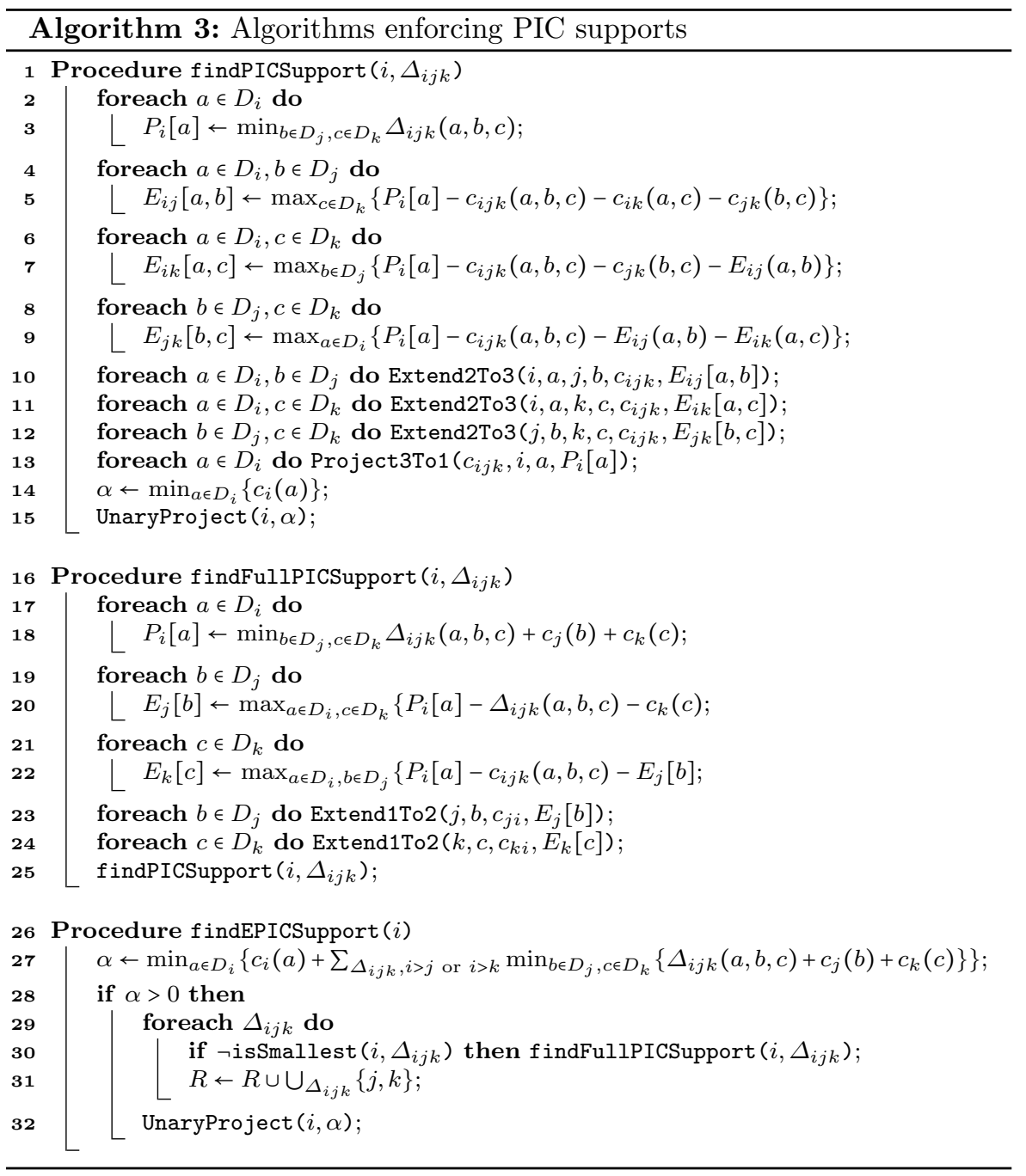

EDPIC is enforced by Procedure enforceEDPIC in Algorithm 4. This procedure consists of four inner-while loops that respectively enforce EPIC, DPIC and PIC. It also enforces NC by calling PruneVars at line 24 .

The first while-loop (lines 5-7) enforces EPIC. It first puts in $R$ all variables that need to be checked for EPIC based on the auxiliary queue $S$ (line 4 ). EPIC supports of variables $i \in R$ are enforced by Procedure findEPICSupport (line 7). When enforcing the existential support for $i$, EPIC is only responsible for triangles on which $i$ is not the smallest variable because DPIC will take care of the remaining ones (Algorithm 3, line 27). If $i$ has no fully supported value (i.e., $\alpha>0$ ) such a value can be created by enforcing full PIC supports for every value of $i$ on every triangle in which $i$ is not the smallest variable 

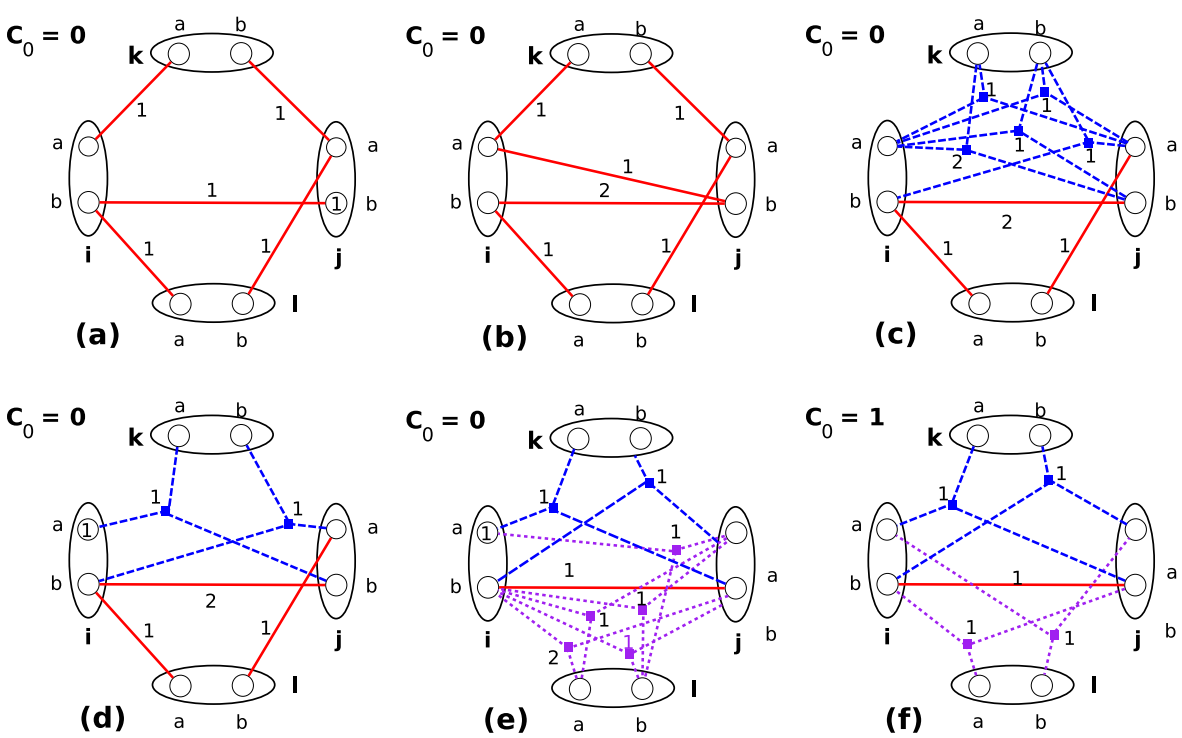

Fig. 14 Cost evolution in a CFN during the enforcement of full PIC supports (a) original problem with 5 binary cost functions $c_{i j}, c_{i k}, c_{i l}, c_{j k}, c_{j l}, i<j<k<l$. It is FDAC but not FDPIC because of variable $i$ where $(i, a)$ and $(i, b)$ cannot be fully extended on $\Delta_{i j k}$ and $\Delta_{i j l}$ respectively. (b) extending a cost of 1 from $j_{b}$ on $c_{i j}$ with $E_{j}[b]=1$. (c) extending a cost of 1 from $\left(i_{a}, j_{b}\right),\left(i_{a}, k_{a}\right)$ and $\left(j_{a}, k_{b}\right)$ on $c_{i j k}$ with $E_{i j}[a, b]=E_{i k}[a, a]=E_{j k}[a, b]=1$. (d) projecting a cost of 1 from $c_{i j k}$ on $i_{a}$ with $P_{i}[a]=1$. (e) extending a cost of 1 from $\left(i_{b}, j_{b}\right),\left(i_{b}, l_{a}\right)$ and $\left(j_{a}, l_{b}\right)$ on $c_{i j k}$ with $E_{i j}[b, b]=E_{i l}[b, a]=E_{j l}[a, b]=1$. (f) projecting a cost of 1 from $c_{i j k}$ on $i_{b}$ with $P_{i}[b]=1$ and then enforcing NC by projecting a cost of 1 from $c_{i}$ on $c_{\varnothing}$. The resulting problem is FDPIC.

(Algorithm 3, line 30). The EPIC supports of neighbor variables of $i$ can also be destroyed (due to new values of non-zero cost made by the enforcement of full PIC supports on $i$ ) and thus are pushed back to $R$ to be later checked for EPIC (Algorithm 3, line 31).

DPIC is enforced by the second while-loop at line 8 . For a variable $j \in P$, only variables that are linked to $j$ by a triangle (line 10) and are the smallest variable of the triangle (lines 11, 12) are considered for checking for DPIC.

PIC is enforced by two while-loops at lines 13 and 18. For a variable $j \in Q$, every neighbor variable of $i$ is checked for PIC. For each $c_{i j} \in T, i, j$ and all variables connected to both $i$ and $j$ are checked for PIC. Simple PIC supports are enforced in the reverse direction of the DAC order, i.e. in triangles in which the considered variables are not the smallest (lines 16-17, 21-23).

From Algorithm 4, algorithms for enforcing other levels of PICs can be obtained by appropriately keeping the right inner while-loops: the first loop (lines 4-7) for EPIC, the second one at line 8 for DPIC, the third one at line 13 for PIC, and three loops at lines 8, 13, 18 for FDPIC. 


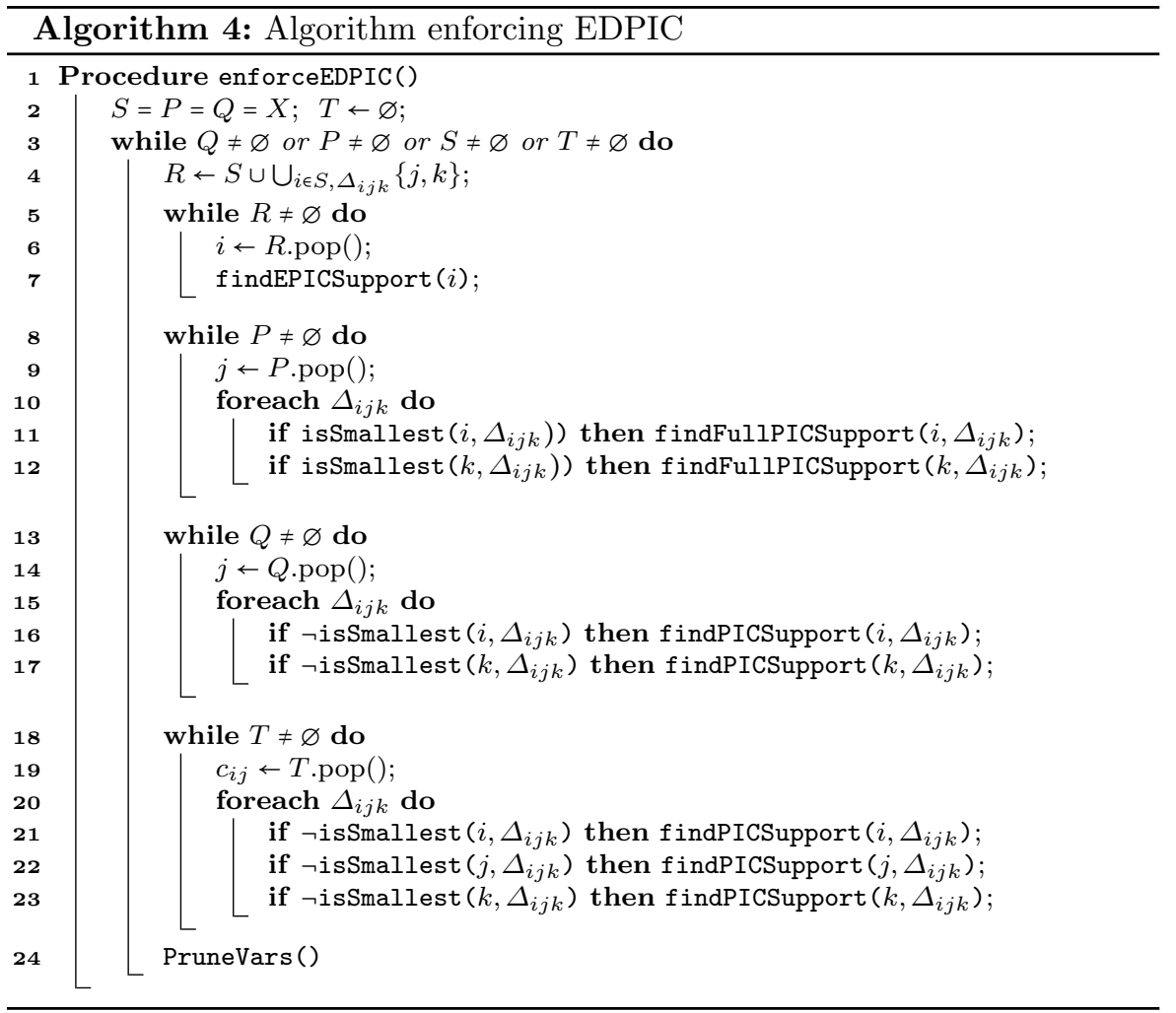

\subsection{Enforcing maxRPCs}

In contrast to PICs that are enforced on triangles sharing a variable, maxRPCs are enforced on triangles sharing two variables of a binary cost function. The extensible arc support of a value $(i, a)$ in a binary cost function $c_{i j}$ is stored in maxRPCSupport $[i, a, j]$ and the witness for this support on a variable $k$ is stored in maxRPCWitness $[i, a, j, k]$. In our algorithms for enforcing soft maxRPCs, we will use a parameter named fullLevel, where fullLevel $=$ false indicates that semi-fully extensible arc supports are used (FDmaxRPC) and fullLevel $=$ true indicates that fully extensible supports are used (EmaxRPC). We will use the following functions:

- $\Lambda_{i j k}(a, b, c)=c_{i k}(a, c)+c_{j k}(b, c)+c_{i j k}(a, b, c)$ : denotes the incompletely combined cost of tuple $(a, b, c)$ (excluding $c_{i j}(a, b)$ from $\Delta_{i j k}(a, b, c)$ ).

- $\Lambda_{i j}^{k}(a, b)=\min _{c \in D_{k}} \Lambda_{i j k}(a, b, c)$ denotes the minimum incompletely combined cost of tuples involving two values $\left(i_{a}, j_{b}\right)$. This is the maximum cost that can be projected on the pair of values $\left(i_{a}, j_{b}\right)$ from two sides $c_{i k}, c_{j k}$ of the triangle $\Delta_{i j k}$ without creating negative costs. 
$\operatorname{argmin} \wedge_{i j}^{k}(a, b)$ is used to denote a value $c \in D_{k}$ for which this minimum is reached. It is a simple witness for the pair $(a, b)$ on the variable $k$.

$-\ddot{\Lambda}_{i j}^{k}(a, b$, fullLevel $)=\left[\begin{array}{ll}\min _{c \in D_{k}}\left\{\Lambda_{i j k}(a, b, c)+c_{k}(c)\right\} & (\text { fullLevel }=\text { true }) \vee(i<k) \\ \min _{c \in D_{k}}\left\{\Lambda_{i j k}(a, b, c)\right\} & \text { (fullLevel }=\text { false) }\end{array}\right]$

is similar to $\Lambda_{i j}^{k}(a, b)$ but it takes into account the unary cost $c_{k}$ of witnesses in the case of (1) fully extensible (fullLevel=true) or (2) semi-fully extensible arc supports on triangles w.r.t DAC order $(i<k)$.

$\operatorname{argmin} \ddot{\wedge}_{i j}^{k}(a, b)$ is used to denote the value $c \in D_{k}$ for which this minimum is reached. It is a (full) witness for the pair $(a, b)$ on the variable $k$.

- $\mathbb{A}_{i j}(a, b)=\sum_{k}\left\{\bigwedge_{i j}^{k}(a, b)\right\}$ is the maximum sum of costs that can be projected on the pair of values $\left(i_{a}, j_{b}\right)$ from all triangles $\Delta_{i j k}$ sharing $i, j$.

- $\ddot{\mathbb{X}}_{i j}(a, b, \mathrm{fullLevel})=\sum_{k}\left\{\ddot{\boldsymbol{X}}_{i j}^{k}(a, b, \mathrm{fullLevel})\right\}$ is similar to $\mathbb{X}_{i j}(a, b)$ but takes into account the unary costs of witnesses $c_{k}$ according to fullLevel and the order between $i$ and $k$ as in the definition of $\ddot{\Lambda}_{i j}^{k}$.

\subsubsection{Enforcing maxRPC supports and witnesses}

Simple maxRPC support for a value $(i, a)$ on $c_{i j}$ is enforced by Procedure findmaxRPCSupport in Algorithm 5. The main idea is to move costs from two sides $c_{i k}, c_{j k}$ of all triangles $\Delta_{i j k}$ to $c_{i j}$ via $c_{i j k}$ (lines 18-20) and finally project costs from $c_{i j}$ to $(i, a)$ (line 21) in such a way that, for each triangle $\Delta_{i j k}$, there exists a value $b \in D_{j}$ and a value $c \in D_{k}$ such that the binary and ternary costs involved in the tuple $\left(i_{a}, j_{b}, k_{c}\right)$ decrease to 0 . The maximum $\operatorname{cost} P_{i}$ that can be projected to $(i, a)$ without creating negative costs is the minimum over all $b \in D_{j}$ of the binary cost of $\left(i_{a}, j_{b}\right)$ that can be obtained by combining the original cost $c_{i j}(a, b)$ and the cost that can be shifted to it from all triangles (computed by function $\mathbb{X}_{i j}$, line 8 ). From this, it becomes possible to compute the actual cost $P_{i j}[a, b]$ that each triangle $\Delta_{i j k}$ provides to $\left(i_{a}, j_{b}\right)$ for this amount of projection to $(i, a)$. It is the minimum of what is needed for this pair of values $\left(P_{i}-c_{i j}(a, b)\right)$ and of what can be provided for it by $\Delta_{i j k}$ (line 13). This condition guarantees that $c_{i j}$ has enough costs to make a unary cost projection $P_{i}$ on $(i, a)$ without creating negative costs. Moreover, if more cost is projected on $c_{i j}$, this cannot lead to a unary cost projection greater than $P_{i}$. In order to project a cost of $P_{i j}[a, b]$ from $c_{i j k}$ to $\left(i_{a}, j_{b}\right)$ (line 20), each side $\left(i_{a}, k_{c}\right)$ and $\left(j_{b}, k_{c}\right)$ has to extend an amount of cost $E_{i k}[a, c]$ and $E_{j k}[b, c]$ to $c_{i j k}$ (lines 19 and 18). These binary cost extensions $E_{i k}[a, c], E_{j k}[b, c]$ are also the minimum of the available cost $c_{i k}(a, c), c_{j k}(b, c)$ that $\left(i_{a}, k_{c}\right),\left(j_{b}, k_{c}\right)$ have and the cost that they need to provide to $c_{i j k}$ (lines 15,17 ).

Full maxRPC supports (covering both fully extensible supports for EmaxRPC and semi-fully extensible for FDmaxRPC) are enforced by findFullmaxRPCSupport in Algorithm 5. The idea for enforcing a full maxRPC support for value $(i, a)$ on $c_{i j}$ is to extend unary costs from $j$ to $c_{i j}$ (line 28) and from third variables $k$ to $c_{i k}$ (line 32 ). Then, costs are moved in the same way as for simple 
maxRPC support in Procedure findmaxRPCSupport (line 33). The maximum cost $P_{i}$ that can be projected on $(i, a)$ is recomputed by taking into account the unary $\operatorname{cost} c_{j}$ of supporting values and the unary $\operatorname{costs} c_{k}$ of witnesses via $\ddot{\mathbb{A}}$ (line 25). In order to achieve this unary projection, each value $(j, b),(k, c)$ needs to extend respectively on $c_{i j}$ and $c_{i k}$ an amount of cost $E_{j}, E_{k}$ (lines 27 and 31). The order in which costs are moved when enforcing full maxRPC supports is described in Figure 15.

An EmaxRPC support for a variable $i$ is enforced thanks to Procedure findEmaxRPCSupport ( $i$ ) in Algorithm 5. It first checks the EmaxRPC property at line 36. If there does not exist any EmaxRPC support (line 37), the procedure will search for a full maxRPC support for any value of $i$ in any cost function $c_{i j}$ by calling findFullmaxRPCSupport with the option fullLevel=true. It only has to take care of the triangles $\Delta_{i j k}$ in which $i$ is the smallest variable, because DmaxRPC takes care of the remaining cases (the condition at line 29 of Procedure findFullmaxRPCSupport).

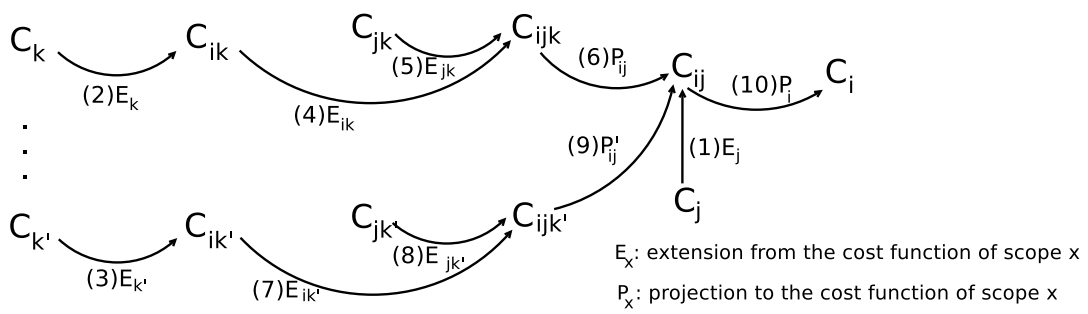

Fig. 15 The order of cost movements for enforcing simple full maxRPC supports where unary cost extensions are not included in the enforcement of simple PIC supports.

Example 3 Consider the CFN(a) in Figure 16. It is FDPIC but not FDmaxRPC because $i_{a}$ has no full AC support in $c_{i j}$ which can be can be extended on both $\Delta_{i j k}$ and $\Delta_{i j l}:\left(i_{a}, j_{a}\right)$ can be extended on $\Delta_{i j l}$ but not on $\Delta_{i j k}$ while $\left(i_{a}, j_{c}\right)$ can be extended on $\Delta_{i j k}$ but $\Delta_{i j l}$. The positive projection/extension costs computed by Procedure findFullmaxRPCSupport $(i, a, j, \mathrm{false})$ are: $P_{i}=$ $2, E_{j}[b]=1$. The procedure extends a cost of 1 from $j_{b}$ on $c_{i j}$ and then calls findmax $R P C(i, a, j)$ which computes the following positive projections/extension costs: $P_{i}=E_{j k}[a, b]=P_{i j}[a, a]=E_{j l}[c, b]=E_{j l}[c, b]=P_{i j}[a, a]=2$. The final problem presented in Sub-figure 16(g) is FDmaxRPC.

Let $j$ be a variable that had a change in the domain $D_{j}$ or in unary cost $c_{j}$ (increasing from 0). The former case can break the witness for simple or semi-full supports of variable $i$ neighbor to $j$ in some $c_{i j}$, while the last case can break the witness for semi-full and full supports. The check and search for new witnesses is performed by Algorithm 6 .

Procedure findWitness_remove $(i, k, j)$ handles the case of domain reduction in $D_{j}$. For any value $(i, a)$, it checks the availability of its current (simple or semi-full) support in $c_{i k}$ (line 5 , algorithm 6 ), as well as the availability of 


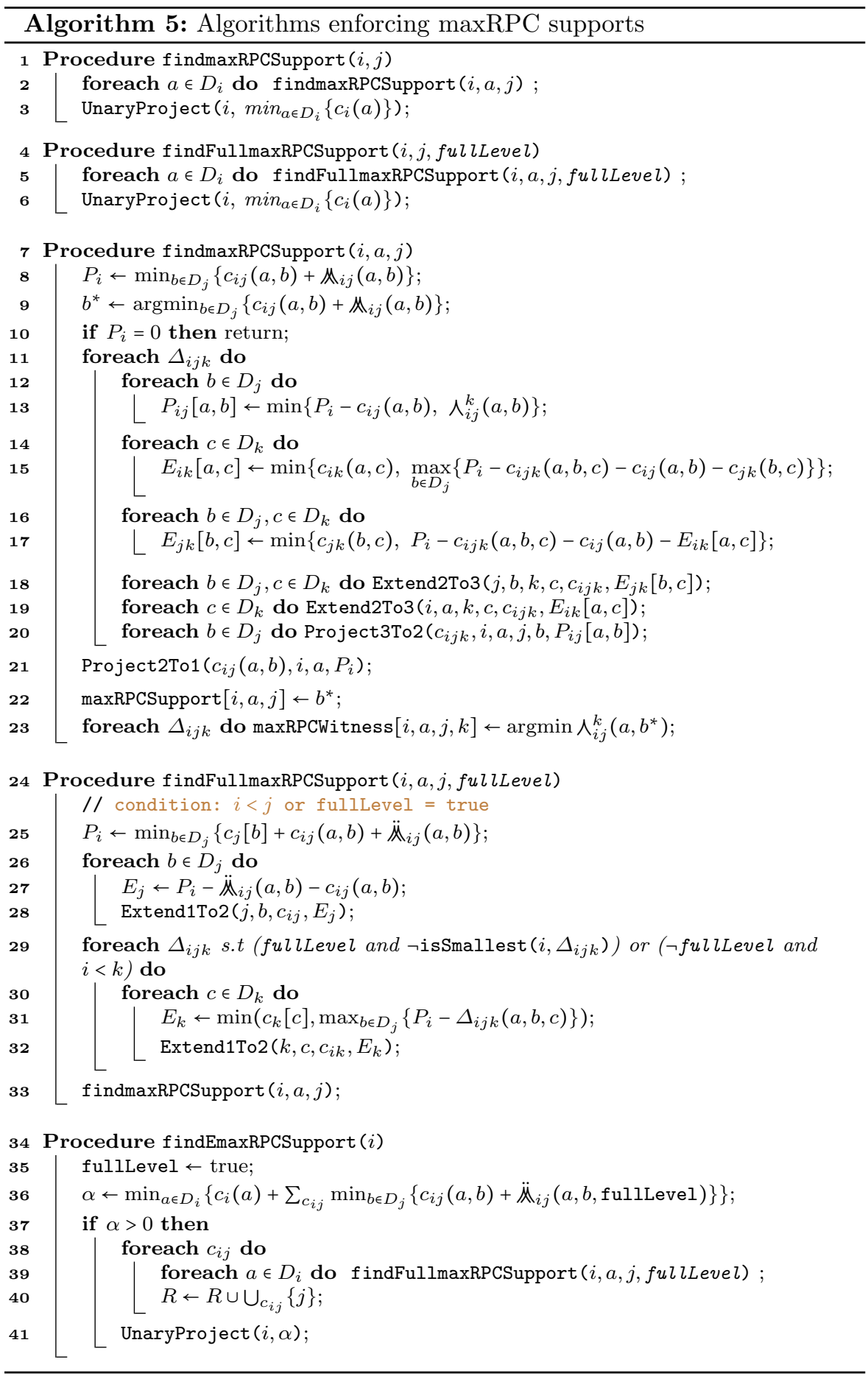




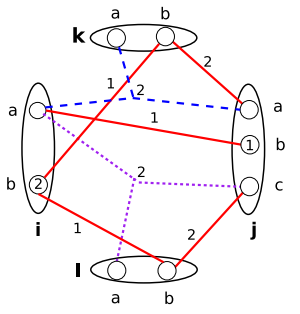

(a) $\mathrm{C}_{0}=\mathrm{o}$

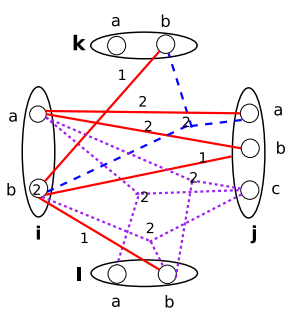

(e) $\mathrm{C}_{0}=\mathbf{0}$

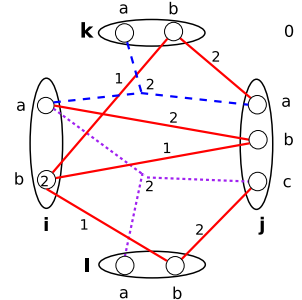

(b) $c_{0}=0$

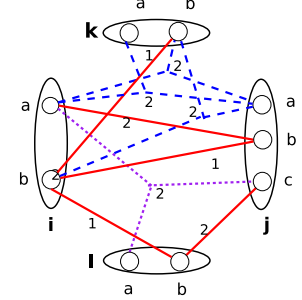

(c) $\mathrm{C}=\mathrm{O}$

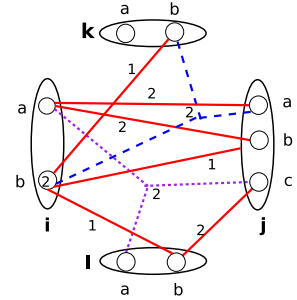

(d) $c_{0}=0$

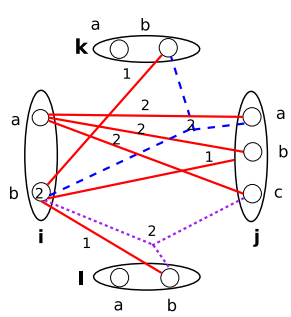

(f) $\mathrm{C}_{0}=\mathbf{0}$

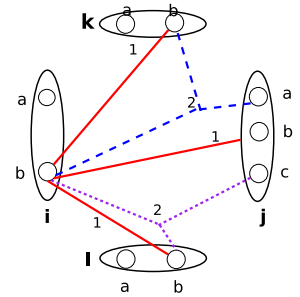

(g) $C_{0}=2$

Fig. 16 Cost evolution during enforcing full maxRPC supports in a CFN (a) original problem with 5 binary cost functions $c_{i j}, c_{i k}, c_{i l}, c_{j k}, c_{j l}$ and 2 ternary functions $c_{i j k}, c_{i j l}$, $i<\{j, k, l\}$. It is FDPIC but not FDmaxRPC due to $i_{a}$ (no full maxRPC support in $c_{i j}$ ) (b) extending a cost of 1 from $j_{b}$ on $c_{i j}$ with $E_{j}[b]=1$ (c) extending a cost of 2 from $\left(j_{a}, k_{b}\right)$ on $c_{i j k}$ with $E_{j k}[a, b]=2(\mathrm{~d})$ projecting a cost of 2 from $c_{i j k}$ on $\left(i_{a}, j_{a}\right)$ with $P_{i j}[a, a]=2$ (e) extending a cost of 2 from $\left(j_{c}, l_{b}\right)$ on $c_{i j k}$ with $E_{j l}[c, b]=2$ (f) projecting a cost of 2 from $c_{i j k}$ on $\left(i_{a}, j_{c}\right)$ with $P_{i j}[a, a]=2$ (g) projecting a cost of 2 from $c_{i j}$ on $i_{a}$ with $P_{i}=2$ and then making $\mathrm{NC}$ by projecting a cost of 2 from $i$ to $c_{\varnothing}$. The resulting problem is FDmaxRPC.

the current witness for this support on $D_{j}$ (line 7 , algorithm 6 ). When the current support has been lost, another support needs to be created for $(i, a)$ (line 11). Similarly, if the current witness is no longer available (line 7), another witness will be searched (line 8). If no witness exists (line 10), another simple or full support needs to be searched for $(i, a)$ according to $i>k$ (line 13) or $i<k$ (line 14) respectively.

Procedure findWitness_project $(i, k, j)$ handles the case where unary $\operatorname{costs} c_{j}$ become positive. This procedure is responsible for semi-full supports as it is only called in the while-loop enforcing DmaxRPC at line 7 of Algorithm 7 . It differs from findWitness_remove() by the fact that unary costs are taken into account when checking the availability of the current supports and witnesses (line 19,21) and when looking for another witness ( $\ddot{\wedge}$ instead of $\wedge$, line 22).

\subsubsection{Soft maxRPC algorithms}

Like PIC, EDmaxRPC includes all softening levels. We thus only present an algorithm for EDmaxRPC. maxRPC, DmaxRPC, FDmaxRPC, and EmaxRPC algorithms can be derived by keeping suitable blocks of code. EDmaxRPC is 


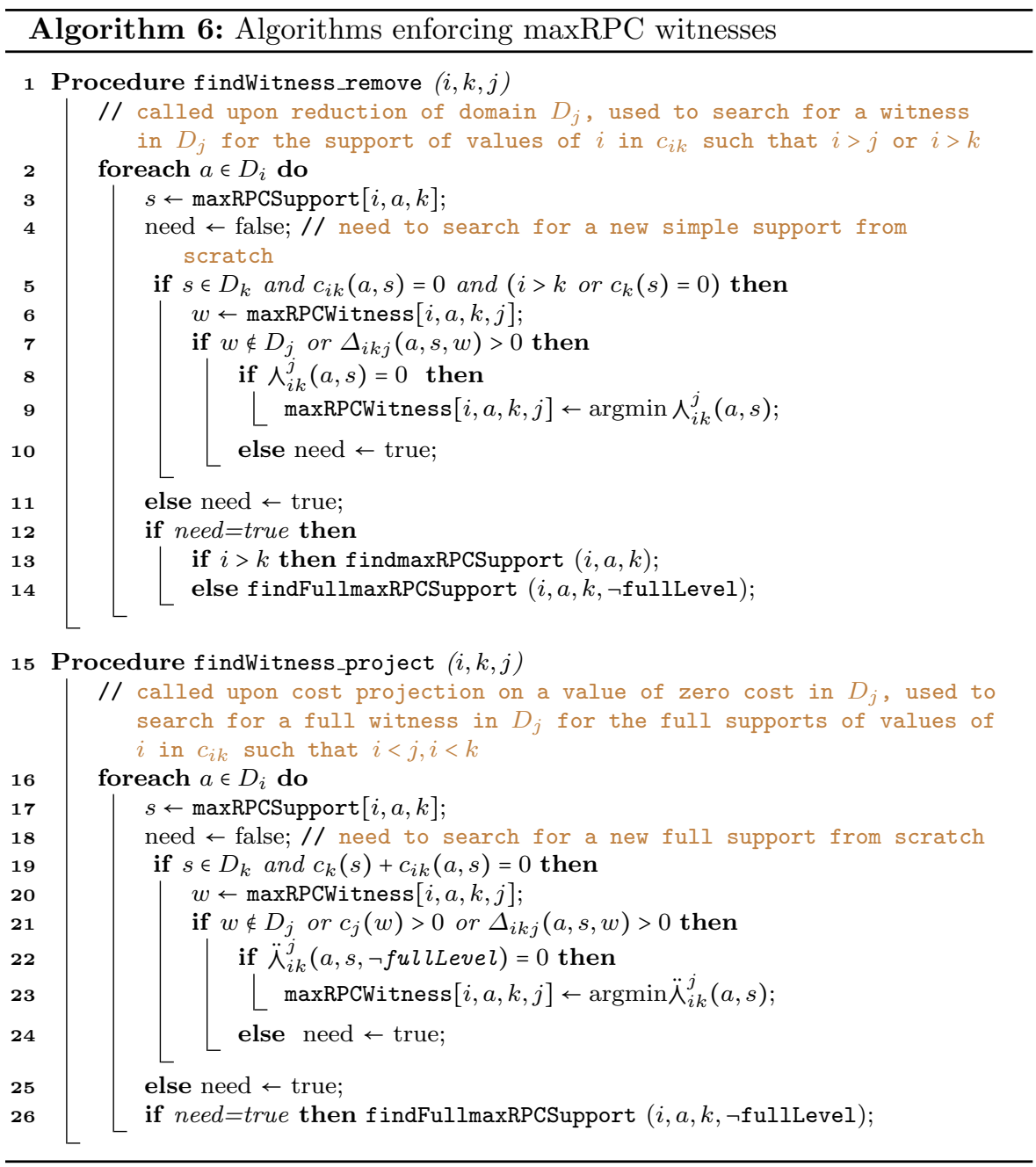

enforced by Procedure enforceEDmaxRPC() in Algorithm 7. It consists of four inner-while loops that handle the same propagation queues $S, P, Q, T$ as in the EDPIC algorithm.

The loop in line 12 enforces maxRPC by propagating domain reductions of $j$ stored in the queue $Q$. For any neighbor value $(i, a)$ of $j$, the deleted values in $D_{j}$ could have been: (1) the simple maxRPC support for $(i, a)$ when $i>j$; (2) the simple witness for the simple maxRPC supports of $(i, a)$ in some $c_{i k}$ when $i>k$; and (3) the simple witness for semi-full maxRPC supports of $(i, a)$ in $c_{i k}$ (of course $i<k$ ) when $i>j$. The deleted values in $D_{j}$ could not have been the full supports and witnesses because they must have a cost of $m$ to be removed. The loop must check and search for (1) a simple maxRPC (line 15-16) and (2) a simple witness (line 17). 


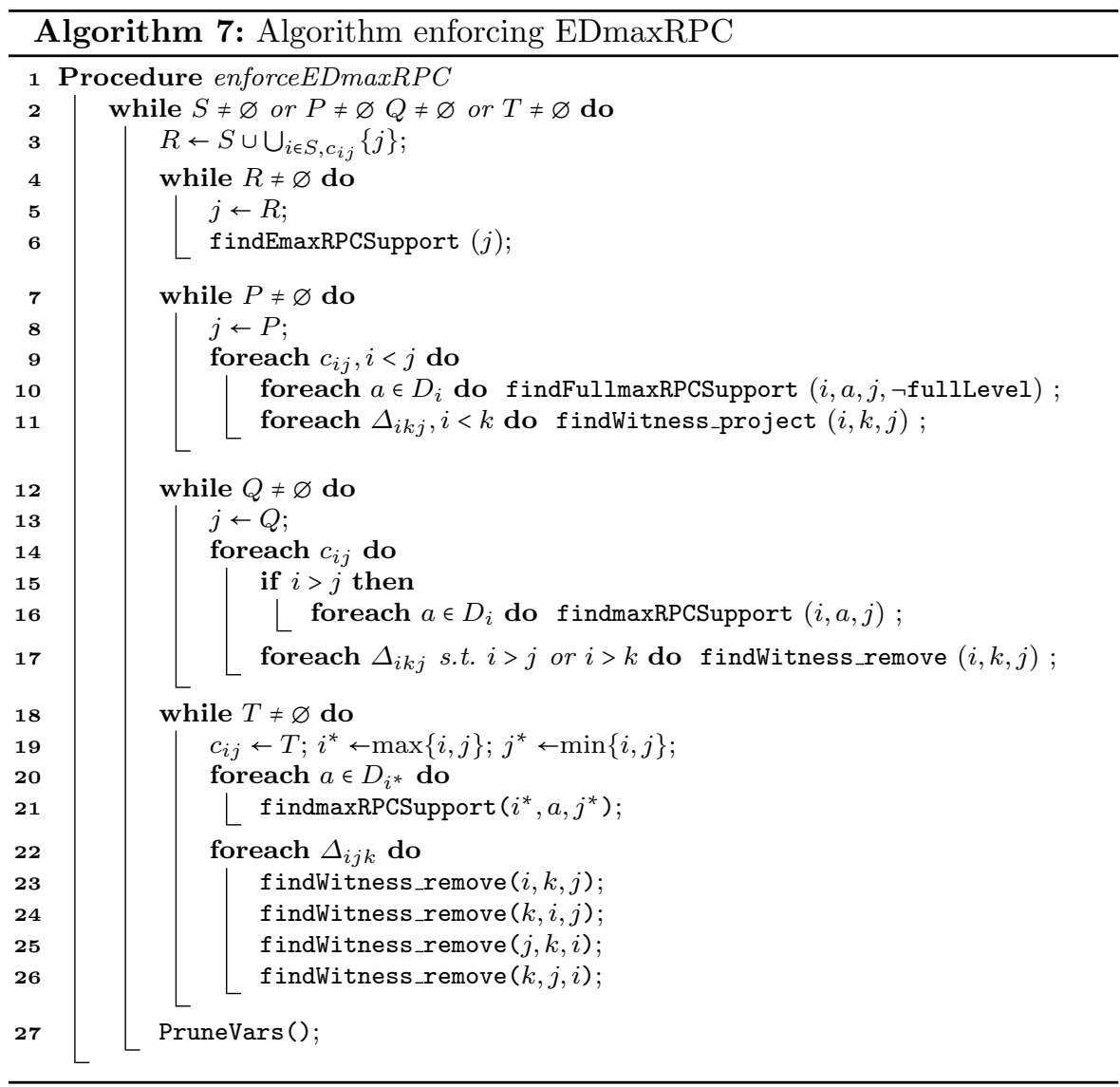

The loop at line 7 enforces DmaxRPC by propagating the increase from 0 of a unary cost $c_{j}$ stored in $P$. The predecessors $i$ of $j$ (such that $c_{i j}$ exists with $i<j$, line 9) may have lost full supports in $c_{i j}$ and thus new full supports need to be searched for values of $i$ (line 10). Moreover, the full supports in $c_{i k}, i<k$ (line 11) can have lost full witnesses on $j$ if $i<j$ (line 9) and thus need to be searched for new witnesses (line 11).

The loop at line 4 enforces EmaxRPC by processing variables in the propagation queue $R$. The construction of $R$ from the auxiliary queue $S$ (line 3 ) is the same as in EDPIC. The search for a EmaxRPC support for a variable $i$ is done by Procedure findEmaxRPCSupport $(i)$ in Algorithm 5.

The loop at line 18 enforces maxRPC by propagating changes in binary $\operatorname{costs} c_{i j}$ (caused by unary cost extensions from the greater variable between $i$ and $j$ on $c_{i j}$ ) stored in queue $T$. Let $i^{*}$ and $j^{*}$ be respectively the greater and the smaller variable between $i$ and $j$. The modified $c_{i j}$ :

- cannot break the full or semi-full maxRPC supports of the smaller variable $j^{*}$ because its values have been supported by values of zero cost. 
- can break the simple maxRPC supports for the values of the greater variable $i^{*}$ and thus new supports need to be searched for such values (line 21).

- can break the witnesses for maxRPC supports in $c_{i k}$ (line 23,24 ) or in $c_{j k}$ (line 25, 26).

From Algorithm 7 enforcing EDmaxRPC, we can obtain algorithms for enforcing other levels of maxRPCs by keeping the first while-loop at line 4 for EmaxRPC, the loop at line 7 for DmaxRPC, the loop at line 12 for maxRPC, and the three loops at lines 7, 12, 18 for FDmaxRPC.

\section{Experimentation}

In this section we provide an experimental evaluation of our soft consistencies. During experimentation, it quickly appeared that maintaining such strong consistencies during search was too time consuming. We therefore decided to relax them in three different ways, denoted as our three use cases.

The three use cases we have considered for our TRICs (triangle-based consistencies) are denoted as:

- TRIC $^{p}$ : uses some TRIC for pre-processing and EDAC during search.

- TRIC $^{r p}$ : uses a restriction of some TRIC, a resTRIC (that will be explained later) for pre-processing, and EDAC during search.

- TRIC $^{r s}$ : uses some resTRIC for both pre-processing and during search.

Restricted TRICs (resTRICs) are defined by limiting the number of triangles to be checked by the consistencies to some maximum. Defining the triangle density of a problem as the ratio of its number of triangles over the number of triangles in a complete graph, we observed that our soft consistencies are often too expensive when used for pre-processing problems having a triangle density larger than $10^{-4}$. We have therefore chosen to bound the number of triangles that are processed in our restricted TRICs to a maximum number $(n(n-1)(n-2) / 6) / 10^{4}$ denoted as $c^{*}$. If $c^{*}<10$, we do not enforce resTRICs and use EDAC only. Otherwise, we bound the number of processed triangles to $c^{*}$. When needed, the triangles chosen to be processed are selected as follows: for each binary cost function $c_{i j}$ we compute its mean cost as $\left(\sum_{a \in D_{i}, b \in D_{j}} c_{i j}(a, b)\right) /\left(\left|D_{i}\right| \times\left|D_{j}\right|\right)$. Triangles are then ranked by decreasing sum of the mean cost of the three involved binary cost functions and the first $c^{*}$ are selected.

In order to evaluate the practical interest of establishing TRICs and their variants, we compared them to the default local consistency enforced in toulbar $2^{3}$ : EDAC. Indeed, EDAC is still the preferred local consistency for Depth First Branch-and-Bound search. We used a large set of benchmarks, as described in Table 1, which has recently been used in [15] for comparing the performance

3 http://mulcyber.toulouse.inra.fr/projects/toulbar2/ version 0.9.6 branch maxrpc. 
of the toulbar2 solver with other solvers ${ }^{4}$. This set consists of the following groups of benchmarks ${ }^{5}$ :

Table 1 The set of benchmarks where each line corresponds to a category of benchmarks (\#inst: number of instances, $\bar{n}$ : mean number of variables, $\bar{d}$ : mean domain size, $\bar{e}$ : mean number of cost functions, $\bar{r}$ : mean arity of cost functions, $\bar{c}$ : mean number of triangles, $\overline{c^{\prime}}$ : mean number of triangles used by TRICs ${ }^{r p}$, and $\overline{\Delta \text { dens: }}$ mean triangle density).

\begin{tabular}{|c|c|c|c|c|c|c|c|c|}
\hline Categories & \#inst & $\bar{n}$ & $\bar{d}$ & $\bar{e}$ & $\bar{r}$ & $\bar{c}$ & $\overline{c^{\prime}}$ & $\overline{\Delta \text { dens }}$ \\
\hline CVPR & 1453 & & & & & & & \\
\hline ChineseChars & 100 & 9147 & 2 & 276677 & 2 & 86557 & 86557 & $1.14 \mathrm{E}-06$ \\
\hline ColorSeg & 21 & 108910 & 9 & 474745 & 2 & 131805 & 32998 & 2.73E-09 \\
\hline GeomSurf-3 & 300 & 505 & 3 & 2140 & 3 & 8 & 8 & 4.46E-07 \\
\hline GeomSurf-7 & 300 & 505 & 7 & 2140 & 3 & 1366 & 1265 & 0.00018 \\
\hline InPainting & 4 & 14400 & 4 & 57121 & 2 & 17732 & 17732 & $3.56 \mathrm{E}-08$ \\
\hline Matching & 4 & 19 & 19 & 166 & 2 & 701 & 0 & 0.679 \\
\hline MatchingSte & 2 & 138407 & 18 & 414477 & 2 & 8 & 8 & $2.70 \mathrm{E}-14$ \\
\hline ObjectSeg & 5 & 68160 & 6 & 203947 & 2 & 31 & 31 & $5.91 \mathrm{E}-13$ \\
\hline PhotoMont & 2 & 469856 & 6 & 1408134 & 2 & 521 & 521 & 4.03E-14 \\
\hline SceneDecp & 715 & 183 & 8 & 672 & 2 & 48 & 42 & $4.80 \mathrm{E}-05$ \\
\hline MaxCSP & 503 & & & & & & & \\
\hline BlackHole & 37 & 114 & 27 & 657 & 2 & 5375 & 38 & 0.01 \\
\hline Coloring & 22 & 120 & 4 & 1323 & 2 & 1227 & 277 & 0.024 \\
\hline Composed & 80 & 58 & 10 & 517 & 2 & 791 & 0 & 0.079 \\
\hline $\mathrm{EHI}$ & 200 & 306 & 7 & 4549 & 2 & 13604 & 475 & 0.0029 \\
\hline Geometric & 100 & 50 & 20 & 471 & 2 & 1694 & 0 & 0.086 \\
\hline Langford & 4 & 25 & 22 & 352 & 2 & 2722 & 0 & 0.736 \\
\hline $\mathrm{QCP}$ & 60 & 159 & 7 & 1384 & 2 & 2671 & 108 & 0.0057 \\
\hline MaxSAT & 427 & & & & & & & \\
\hline Haplotyping & 100 & 150428 & 2 & 534105 & 483 & 61646 & 61646 & $2.39 \mathrm{E}-10$ \\
\hline MaxClique & 62 & 484 & 2 & 50093 & 2 & 1070886 & 2019 & 0.079 \\
\hline MIPLib & 12 & 10523 & 2 & 45991 & 20 & 104 & 104 & $5.92 \mathrm{E}-07$ \\
\hline PackupWei & 99 & 9492 & 2 & 23731 & 61 & 9236 & 9236 & $6.87 \mathrm{E}-07$ \\
\hline PlanWithPre & 29 & 14991 & 2 & 111259 & 64 & 8026 & 8026 & $1.76 \mathrm{E}-06$ \\
\hline TimeTabling & 25 & 128243 & 2 & 785222 & 21 & 40052 & 40052 & $1.58 \mathrm{E}-09$ \\
\hline Upgrad & 100 & 18169 & 2 & 105097 & 77 & 1884 & 1884 & $1.88 \mathrm{E}-09$ \\
\hline UAI & 211 & & & & & & & \\
\hline Grid & 21 & 3143 & 2 & 9379 & 2 & 2 & 2 & $3.74 \mathrm{E}-08$ \\
\hline ImageAlign & 10 & 191 & 70 & 1819 & 2 & 6218 & 37 & 0.0058 \\
\hline Linkage & 22 & 917 & 5 & 1560 & 4 & 13 & 13 & $2.23 \mathrm{E}-07$ \\
\hline ObjDetect & 37 & 60 & 17 & 1830 & 2 & 34220 & 0 & 1 \\
\hline ProteinFold & 21 & 486 & 267 & 2291 & 2 & 4698 & 273 & 0.52 \\
\hline Segment & 100 & 229 & 12 & 851 & 2 & 315 & 185 & 0.00016 \\
\hline CFN & 226 & & & & & & & \\
\hline Auction & 170 & 140 & 2 & 3593 & 2 & 47707 & 57 & 0.0869 \\
\hline CELAR & 16 & 126 & 44 & 641 & 2 & 837 & 46 & 0.228 \\
\hline Pedigree & 10 & 1758 & 11 & 3247 & 3 & 70 & 70 & $3.96 \mathrm{E}-06$ \\
\hline ProteinDsn & 10 & 13 & 123 & 97 & 2 & 311 & 0 & 0.966 \\
\hline SPOT5 & 20 & 385 & 4 & 6603 & 3 & 35976 & 2900 & 0.0055 \\
\hline
\end{tabular}

${ }^{4}$ We only excluded from the set all 35 Minizinc instances as well as two subcategories (UAI/DBN, 108 instances and CSP/warehouse, 55 instances) that contain no triangle of binary cost functions. Over the original 3,018 original instances, 2,820 remain.

5 All the instances are available at http://genoweb.toulouse.inra.fr/ degivry/evalgm. 
- WCSP: contains cost function networks extracted from the Cost Function Library $^{6}$, including Combinatorial Auctions [18], Radio Link Frequency Assignment problems [4], Mendelian error correction problems on complex pedigree [23], Computational Protein Design problems [1] and SPOT5 satellite scheduling problems [2].

- MaxCSP ${ }^{7}$ : contains unsatisfiable binary CSP instances with constraints defined in extension, including BlackHole, Langford, Quasi-group completion problem,graph coloring, random composed, and random Geometric.

- UAI: consists of Markov Random Field problems that are collected from the Probabilistic Inference Challenge $2011^{8}$ and Genetic Linkage Analysis problems[13].

- MaxSAT: contains Max-SAT instances that are collected from the MaxSAT Evaluation ${ }^{9}$.

- CVPR: contains MRF instances from the Computer Vision and Pattern Recognition (CVPR) OpenGM2 benchmark ${ }^{10}$.

Our algorithms were all implemented with a time-limit after which we consider the instance as not solved by this algorithm. For all categories of instances except ChineseChars and GeomSurf7, the time-limit was set to 1200 seconds. For ChineseChars and GeomSurf7 we set the time-limit to 3600 seconds as the instances in these categories were significantly harder.

\section{Number of solved instances}

Table 2 reports the number of instances per category of benchmarks that are solved by a Depth-First Branch\&Bound algorithm using each consistency (EDAC and TRICs implemented in the three use cases). The first block of three lines reports the total number of instances solved by each algorithm in all categories. The remaining lines focus on selected categories where a difference in behavior was observed. These results show that in general $\mathrm{TRIC}^{r p}$ is the best choice, independently of the chosen triangle consistency. It however works best in combination with the strongest EDmaxRPC consistency. Then EDAC, $\mathrm{TRIC}^{r s}$, and finally $\mathrm{TRIC}^{p}$ follow. One can observe that for the three use cases, TRICs (especially EDmaxRPC) are very efficient on the ChineseChars and GeomSurf7 problems, which are defined on grid graphs. While EDAC cannot solve any ChineseChars instance (this is also the case for all the other solvers reported in [15], including ILP and toulbar2 using VAC), TRICs can solve a certain number of instances ( 8 for PIC and 16 EDmaxRPC). Similarly, TRICs can solve up to $5 \%$ instances more than EDAC on GeomSurf-7. The advantage of TRICs on these problems are more clearly shown in Table 3 : many instances cannot be solved in 1 hour by maintaining EDAC but can be solved by TRICs in less than 100 seconds.

\footnotetext{
6 https://mulcyber.toulouse.inra.fr/scm/viewvc.php/trunk/?root=costfunctionlib

7 http://www.cril.univ-artois.fr/CPAI08/, ../ lecoutre/benchmarks.html

8 http://www.cs.huji.ac.il/project/PASCAL/realBoard.php

9 http://maxsat.ia.udl.cat:81/13/benchmarks/

10 http://hci.iwr.uni-heidelberg.de/opengm2
} 
On the rest of the benchmarks, especially on categories having a very large mean triangle density such as Geometric, MaxClique, ProteinFold, Auction and CELAR (respectively 0.086, 0.079, 0.52, 0.0869 and 0.228), $\mathrm{TRIC}^{p}$ becomes worse than EDAC and solves 5,5\%,64\%, 47\%, 25\% and $75 \%$ less instances respectively. The same behavior is observed on PackupWei (decrease by 11,5\%). For these problems, the restricted versions $\mathrm{TRIC}^{r p}$ can significantly improve the efficiency of $\mathrm{TRIC}^{p}$ and give results comparable to EDAC, thanks to the reduction in the number of triangles processed. In all cases, $\mathrm{TRIC}^{r s}$ are less efficient than $\mathrm{TRIC}^{r p}$.

Table 2: The number of instances per category solved in less than 1200 seconds (1 hour for the CVPR group). Each block corresponds to a category of benchmarks whose name and size are given in the two first columns. The number of instances per category solved by EDAC and TRICs are respectively given in the 3rd and the 8 last columns. Three lines of the combined blocks correspond respectively to the three use cases: $\mathrm{TRICs}^{p}, \mathrm{TRICs}^{r p}$ and $\mathrm{TRICs}^{r s}$. For the categories absent from the table, $\mathrm{TRICs}^{p}, \mathrm{TRICs}^{r p}$ and $\mathrm{TRICs}^{r s}$ give the same result as EDAC. Best results are in bold.

\begin{tabular}{|c|c|c|c|c|c|c|c|c|c|c|c|}
\hline Problems & inst & & 㽦 & $\stackrel{\circlearrowright}{\Omega}$ & $\frac{\circlearrowright}{\stackrel{0}{a}}$ & 它 & $\stackrel{\circlearrowright}{\stackrel{\varrho}{a}}$ & 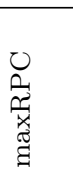 & 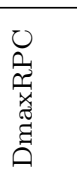 & 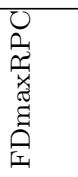 & 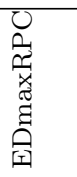 \\
\hline Summary & 2820 & $\begin{array}{c}\mathrm{p} \\
\mathrm{rp} \\
\mathrm{rs}\end{array}$ & 2053 & $\begin{array}{l}1972 \\
2051 \\
2013\end{array}$ & $\begin{array}{l}1980 \\
2055 \\
2031\end{array}$ & $\begin{array}{l}1979 \\
2060 \\
2030\end{array}$ & $\begin{array}{l}1979 \\
2058 \\
2018\end{array}$ & $\begin{array}{l}1967 \\
2059 \\
1993\end{array}$ & $\begin{array}{l}1982 \\
2069 \\
2010\end{array}$ & $\begin{array}{l}1980 \\
2072 \\
1992\end{array}$ & $\begin{array}{l}1981 \\
\mathbf{2 0 7 4} \\
1998\end{array}$ \\
\hline ChineseChars & 100 & $\begin{array}{l}\mathrm{p} \\
\mathrm{rp} \\
\mathrm{rs}\end{array}$ & 0 & $\begin{array}{l}8 \\
9 \\
9\end{array}$ & $\begin{array}{l}8 \\
7 \\
9\end{array}$ & $\begin{array}{l}10 \\
9 \\
10\end{array}$ & $\begin{array}{l}10 \\
10 \\
10\end{array}$ & $\begin{array}{l}10 \\
14 \\
11\end{array}$ & $\begin{array}{l}9 \\
10 \\
10\end{array}$ & $\begin{array}{l}10 \\
13 \\
12\end{array}$ & $\begin{array}{l}\mathbf{1 6} \\
15 \\
11\end{array}$ \\
\hline GeomSurf-7 & 300 & $\begin{array}{l}\mathrm{p} \\
\mathrm{rp} \\
\mathrm{rs}\end{array}$ & 281 & $\begin{array}{l}280 \\
280 \\
278\end{array}$ & $\begin{array}{l}287 \\
284 \\
283\end{array}$ & $\begin{array}{l}285 \\
288 \\
289\end{array}$ & $\begin{array}{l}288 \\
290 \\
287\end{array}$ & $\begin{array}{l}281 \\
280 \\
273\end{array}$ & $\begin{array}{l}292 \\
292 \\
285\end{array}$ & $\begin{array}{l}292 \\
292 \\
281\end{array}$ & $\begin{array}{l}\mathbf{2 9 5} \\
294 \\
282\end{array}$ \\
\hline Coloring & 22 & $\begin{array}{l}\mathrm{p} \\
\mathrm{rp} \\
\mathrm{rs}\end{array}$ & 17 & $\begin{array}{l}\mathbf{1 8} \\
17 \\
17\end{array}$ & $\begin{array}{l}\mathbf{1 8} \\
17 \\
17\end{array}$ & $\begin{array}{l}17 \\
17 \\
16\end{array}$ & $\begin{array}{l}17 \\
17 \\
16\end{array}$ & $\begin{array}{l}\mathbf{1 8} \\
17 \\
17\end{array}$ & $\begin{array}{l}\mathbf{1 8} \\
17 \\
16\end{array}$ & $\begin{array}{l}\mathbf{1 8} \\
17 \\
16\end{array}$ & $\begin{array}{l}\mathbf{1 8} \\
17 \\
16\end{array}$ \\
\hline Geometric & 100 & $\begin{array}{l}\mathrm{p} \\
\mathrm{rp} \\
\mathrm{rs}\end{array}$ & 91 & $\begin{array}{l}88 \\
92 \\
90 \\
\end{array}$ & $\begin{array}{l}87 \\
91 \\
90 \\
\end{array}$ & $\begin{array}{l}87 \\
92 \\
90 \\
\end{array}$ & $\begin{array}{l}86 \\
92 \\
86 \\
\end{array}$ & $\begin{array}{l}87 \\
92 \\
87 \\
\end{array}$ & $\begin{array}{l}87 \\
\mathbf{9 3} \\
87 \\
\end{array}$ & $\begin{array}{l}86 \\
91 \\
88 \\
\end{array}$ & $\begin{array}{l}86 \\
92 \\
86 \\
\end{array}$ \\
\hline $\mathrm{QCP}$ & 60 & $\begin{array}{l}\mathrm{p} \\
\mathrm{rp} \\
\mathrm{rs}\end{array}$ & 14 & $\begin{array}{l}14 \\
14 \\
14\end{array}$ & $\begin{array}{l}14 \\
14 \\
14\end{array}$ & $\begin{array}{l}14 \\
14 \\
14\end{array}$ & $\begin{array}{l}14 \\
14 \\
14\end{array}$ & $\begin{array}{l}14 \\
14 \\
13\end{array}$ & $\begin{array}{l}14 \\
14 \\
13\end{array}$ & $\begin{array}{l}14 \\
14 \\
13\end{array}$ & $\begin{array}{l}14 \\
14 \\
14\end{array}$ \\
\hline Haplotyping & 100 & $\begin{array}{l}\mathrm{p} \\
\mathrm{rp} \\
\mathrm{rs}\end{array}$ & 1 & $\begin{array}{l}1 \\
1 \\
1 \\
\end{array}$ & $\begin{array}{l}1 \\
1 \\
1\end{array}$ & $\begin{array}{l}1 \\
1 \\
1 \\
\end{array}$ & $\begin{array}{l}1 \\
1 \\
1\end{array}$ & $\begin{array}{l}1 \\
2 \\
1\end{array}$ & $\begin{array}{l}\mathbf{2} \\
\mathbf{2} \\
1 \\
\end{array}$ & $\begin{array}{l}\mathbf{2} \\
\mathbf{2} \\
1 \\
\end{array}$ & $\begin{array}{l}1 \\
\mathbf{2} \\
1 \\
\end{array}$ \\
\hline MaxClique & 62 & $\begin{array}{l}\mathrm{p} \\
\mathrm{rp} \\
\mathrm{rs}\end{array}$ & 33 & $\begin{array}{l}15 \\
28 \\
24\end{array}$ & $\begin{array}{l}14 \\
29 \\
27\end{array}$ & $\begin{array}{l}15 \\
30 \\
24\end{array}$ & $\begin{array}{l}14 \\
29 \\
26\end{array}$ & $\begin{array}{l}13 \\
29 \\
23\end{array}$ & $\begin{array}{l}12 \\
29 \\
23\end{array}$ & $\begin{array}{l}14 \\
30 \\
22\end{array}$ & $\begin{array}{l}13 \\
30 \\
22\end{array}$ \\
\hline MIPLib & 12 & $\begin{array}{c}\mathrm{p} \\
\mathrm{rp} \\
\mathrm{rs}\end{array}$ & 3 & $\begin{array}{l}\mathbf{3} \\
\mathbf{3} \\
2 \\
\end{array}$ & $\begin{array}{l}\mathbf{3} \\
\mathbf{3} \\
2\end{array}$ & $\begin{array}{l}\mathbf{3} \\
\mathbf{3} \\
2 \\
\end{array}$ & $\begin{array}{l}\mathbf{3} \\
\mathbf{3} \\
2 \\
\end{array}$ & $\begin{array}{l}\mathbf{3} \\
\mathbf{3} \\
2 \\
\end{array}$ & $\begin{array}{l}\mathbf{3} \\
\mathbf{3} \\
2 \\
\end{array}$ & $\begin{array}{l}\mathbf{3} \\
\mathbf{3} \\
2\end{array}$ & $\begin{array}{l}\mathbf{3} \\
\mathbf{3} \\
2\end{array}$ \\
\hline PackupWei & 99 & $\begin{array}{l}\mathrm{p} \\
\mathrm{rp} \\
\mathrm{rs}\end{array}$ & 52 & $\begin{array}{l}48 \\
48 \\
41 \\
\end{array}$ & $\begin{array}{l}47 \\
46 \\
39 \\
\end{array}$ & $\begin{array}{l}47 \\
48 \\
42 \\
\end{array}$ & $\begin{array}{l}47 \\
47 \\
40\end{array}$ & $\begin{array}{l}47 \\
48 \\
41 \\
\end{array}$ & $\begin{array}{l}46 \\
47 \\
39 \\
\end{array}$ & $\begin{array}{l}47 \\
47 \\
40\end{array}$ & $\begin{array}{l}47 \\
47 \\
40\end{array}$ \\
\hline Upgrad & 100 & $\mathrm{p}$ & 100 & 100 & 100 & 100 & 98 & 100 & 100 & 99 & 98 \\
\hline
\end{tabular}




\begin{tabular}{|c|c|c|c|c|c|c|c|c|c|c|c|}
\hline Problems & inst & & $\underset{\Theta}{己}$ & $\frac{0}{2}$ & $\frac{\circlearrowright}{\overbrace{1}}$ & 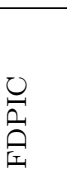 & $\begin{array}{l}\circlearrowright \\
\stackrel{0}{D_{1}} \\
\stackrel{1}{|c|}\end{array}$ & 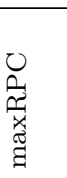 & 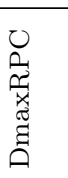 & 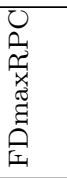 & 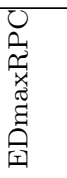 \\
\hline & & $\begin{array}{l}\text { rp } \\
\text { rs }\end{array}$ & & $\begin{array}{l}96 \\
92\end{array}$ & $\begin{array}{l}100 \\
100\end{array}$ & $\begin{array}{l}96 \\
94\end{array}$ & $\begin{array}{l}92 \\
92\end{array}$ & $\begin{array}{l}97 \\
89\end{array}$ & $\begin{array}{l}99 \\
96\end{array}$ & $\begin{array}{l}98 \\
93\end{array}$ & $\begin{array}{l}97 \\
91\end{array}$ \\
\hline ImageAlign & 10 & $\begin{array}{l}\mathrm{p} \\
\mathrm{rp} \\
\mathrm{rs}\end{array}$ & 10 & $\begin{array}{l}7 \\
10 \\
10\end{array}$ & $\begin{array}{l}9 \\
10 \\
10\end{array}$ & $\begin{array}{l}7 \\
10 \\
10\end{array}$ & $\begin{array}{l}7 \\
10 \\
10\end{array}$ & $\begin{array}{l}6 \\
\mathbf{1 0} \\
\mathbf{1 0}\end{array}$ & $\begin{array}{l}7 \\
10 \\
10\end{array}$ & $\begin{array}{l}5 \\
10 \\
10\end{array}$ & $\begin{array}{l}5 \\
10 \\
10\end{array}$ \\
\hline Linkage & 22 & $\begin{array}{l}\mathrm{p} \\
\mathrm{rp} \\
\mathrm{rs}\end{array}$ & 13 & $\begin{array}{l}13 \\
14 \\
11\end{array}$ & $\begin{array}{l}13 \\
13 \\
10\end{array}$ & $\begin{array}{l}13 \\
14 \\
11\end{array}$ & $\begin{array}{l}14 \\
14 \\
10\end{array}$ & $\begin{array}{l}14 \\
14 \\
9\end{array}$ & $\begin{array}{l}13 \\
14 \\
10\end{array}$ & $\begin{array}{l}\mathbf{1 5} \\
\mathbf{1 5} \\
10\end{array}$ & $\begin{array}{l}\mathbf{1 5} \\
\mathbf{1 5} \\
9\end{array}$ \\
\hline ProteinFold & 21 & $\begin{array}{l}\mathrm{p} \\
\mathrm{rp} \\
\mathrm{rs}\end{array}$ & 19 & $\begin{array}{l}10 \\
20 \\
20\end{array}$ & $\begin{array}{l}10 \\
20 \\
20\end{array}$ & $\begin{array}{l}10 \\
20 \\
20\end{array}$ & $\begin{array}{l}10 \\
20 \\
20\end{array}$ & $\begin{array}{l}10 \\
20 \\
20\end{array}$ & $\begin{array}{l}10 \\
20 \\
20\end{array}$ & $\begin{array}{l}10 \\
\mathbf{2 0} \\
19\end{array}$ & $\begin{array}{l}10 \\
20 \\
20\end{array}$ \\
\hline Segment & 100 & $\begin{array}{l}\mathrm{p} \\
\mathrm{rp} \\
\mathrm{rs}\end{array}$ & 100 & $\begin{array}{l}\mathbf{1 0 0} \\
99 \\
98\end{array}$ & $\begin{array}{l}100 \\
100 \\
100\end{array}$ & $\begin{array}{l}\mathbf{1 0 0} \\
\mathbf{1 0 0} \\
99\end{array}$ & $\begin{array}{l}\mathbf{1 0 0} \\
\mathbf{1 0 0} \\
98\end{array}$ & $\begin{array}{l}\mathbf{1 0 0} \\
99 \\
98\end{array}$ & $\begin{array}{l}\mathbf{1 0 0} \\
99 \\
\mathbf{1 0 0}\end{array}$ & $\begin{array}{l}\mathbf{1 0 0} \\
\mathbf{1 0 0} \\
98\end{array}$ & $\begin{array}{l}\mathbf{1 0 0} \\
\mathbf{1 0 0} \\
98\end{array}$ \\
\hline Auction & 170 & $\begin{array}{l}\mathrm{p} \\
\mathrm{rp} \\
\mathrm{rs}\end{array}$ & 166 & $\begin{array}{l}126 \\
\mathbf{1 6 7} \\
154\end{array}$ & $\begin{array}{l}128 \\
\mathbf{1 6 7} \\
156\end{array}$ & $\begin{array}{l}130 \\
166 \\
156\end{array}$ & $\begin{array}{l}129 \\
\mathbf{1 6 7} \\
154\end{array}$ & $\begin{array}{l}125 \\
\mathbf{1 6 7} \\
147\end{array}$ & $\begin{array}{l}129 \\
\mathbf{1 6 7} \\
146\end{array}$ & $\begin{array}{l}129 \\
\mathbf{1 6 7} \\
146\end{array}$ & $\begin{array}{l}125 \\
165 \\
144\end{array}$ \\
\hline CELAR & 16 & $\begin{array}{l}\mathrm{p} \\
\mathrm{rp} \\
\mathrm{rs}\end{array}$ & 12 & $\begin{array}{l}4 \\
\mathbf{1 2} \\
11\end{array}$ & $\begin{array}{l}4 \\
12 \\
12\end{array}$ & $\begin{array}{l}3 \\
11 \\
11\end{array}$ & $\begin{array}{l}3 \\
11 \\
11\end{array}$ & $\begin{array}{l}3 \\
\mathbf{1 2} \\
11\end{array}$ & $\begin{array}{l}3 \\
\mathbf{1 2} \\
11\end{array}$ & $\begin{array}{l}3 \\
\mathbf{1 2} \\
11\end{array}$ & $\begin{array}{l}3 \\
\mathbf{1 2} \\
11\end{array}$ \\
\hline Matching & 4 & $\begin{array}{l}\mathrm{p} \\
\mathrm{rp} \\
\mathrm{rs}\end{array}$ & 4 & $\begin{array}{l}4 \\
4 \\
4\end{array}$ & $\begin{array}{l}4 \\
4 \\
4\end{array}$ & $\begin{array}{l}4 \\
4 \\
4\end{array}$ & $\begin{array}{l}4 \\
4 \\
4\end{array}$ & $\begin{array}{l}2 \\
4 \\
4\end{array}$ & $\begin{array}{l}4 \\
4 \\
4\end{array}$ & $\begin{array}{l}0 \\
4 \\
4\end{array}$ & $\begin{array}{l}0 \\
4 \\
4\end{array}$ \\
\hline ProteinDsn & 10 & $\begin{array}{l}\mathrm{p} \\
\mathrm{rp} \\
\mathrm{rs}\end{array}$ & 9 & $\begin{array}{l}5 \\
9 \\
9\end{array}$ & $\begin{array}{l}5 \\
9 \\
9\end{array}$ & $\begin{array}{l}5 \\
9 \\
9\end{array}$ & $\begin{array}{l}5 \\
9 \\
9\end{array}$ & $\begin{array}{l}5 \\
9 \\
9\end{array}$ & $\begin{array}{l}5 \\
9 \\
9\end{array}$ & $\begin{array}{l}5 \\
9 \\
9\end{array}$ & $\begin{array}{l}4 \\
\mathbf{9} \\
\mathbf{9}\end{array}$ \\
\hline
\end{tabular}

In Figure 17 we present a cactus plot over all instances of all categories. This gives us an overall view on the performance of our 18 TRICs and EDAC. This cactus plot makes obvious that $\mathrm{TRICs}^{r p}$ are consistently the best while $\mathrm{TRICs}^{p}$ are the worst, not only in terms of the number of solved instances, as shown in Table 2, but also in terms of running time. EDAC is ranked somewhere among the worst of the TRICs ${ }^{r p}$ use case. EDmaxRPC ${ }^{r p}$, FDmaxRPC $^{r p}$, and DmaxRPC ${ }^{r p}$ are the three algorithms that seem to be the more reliable, being among the best whatever the time allowed. Surprisingly, there is no consistency that clearly outperforms the others on all use cases.

The apparent dominance of $\mathrm{TRICS}^{r p}$ over $\mathrm{TRICS}^{p}$ is also the direct consequence of the presence of a number of relatively easy instances in our set of benchmark problems. As we have seen, on families of hard instances, TRICS $^{p}$ can be more efficient than TRICS ${ }^{r p}$.

\section{Number of backtracks}

Figure 18 presents the mean number of backtracks, computed over all instances that could be solved by all approaches, for EDAC and the three use cases of TRICs. It shows that the number of backtracks is consistent with the 
Table 3 Solving time (in seconds) for maintaining EDAC and using TRICs ${ }^{p}$ for a subset of benchmarks. This subset contains only ChineseChars and GeomSurf- 7 instances that respectively can and cannot be solved by one of the consistencies in 1 hour. "-" means that the problem cannot be solved. Best results are in bold.

\begin{tabular}{|c|c|c|c|c|c|c|c|c|c|}
\hline Problem & 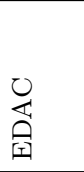 & $O_{1}$ & $\frac{0}{2}$ & 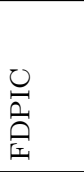 & $\begin{array}{l}0 \\
\stackrel{0}{\Lambda_{1}} \\
\text { 至 }\end{array}$ & 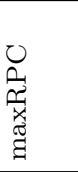 & 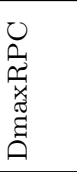 & 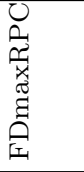 & 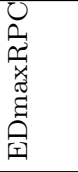 \\
\hline \multicolumn{10}{|c|}{ ChineseChars (TST_) } \\
\hline$\overline{0012 \_88 \_103}$ & - & 195 & 575 & 34 & 41 & 21 & 119 & 27 & 44 \\
\hline 0020_96_94 & - & - & 2647 & 249 & 260 & 363 & 1709 & 158 & 158 \\
\hline 0024_88_126 & - & - & - & - & - & - & - & - & 662 \\
\hline 0027_88_109 & - & - & - & - & - & - & - & - & 865 \\
\hline 0041_88_96 & - & - & - & 3149 & 1569 & 269 & - & 760 & 114 \\
\hline 0047_112_121 & - & 215 & 83 & 23 & 49 & 18 & 26 & 28 & 64 \\
\hline 0052_96_107 & - & 1230 & 3564 & 1842 & 154 & 77 & 690 & 183 & 46 \\
\hline 0059_104_73 & - & 130 & 93 & 37 & 32 & 11 & 28 & 20 & 33 \\
\hline 0067_96_121 & - & 201 & 590 & 117 & 143 & 47 & 151 & 41 & 76 \\
\hline 0070_88_96 & - & 460 & 2194 & 392 & 158 & 56 & 210 & 99 & 73 \\
\hline 0084_120_115 & - & - & - & - & - & - & - & - & 416 \\
\hline 0087_88_124 & - & - & - & - & - & - & - & - & 1910 \\
\hline 0089_72_92 & - & - & - & - & - & - & - & - & 1148 \\
\hline 0099_72_105 & - & 502 & 2012 & 112 & 101 & 30 & 347 & 65 & 75 \\
\hline 0100_80_102 & - & 1199 & - & 591 & 532 & 227 & 1577 & 402 & 78 \\
\hline \multicolumn{10}{|l|}{ GeomSurf-7 } \\
\hline gm113 & 1487 & - & 349 & 254 & 486 & 678 & 166 & 95 & 138 \\
\hline gm125 & - & - & 1877 & 2938 & - & - & 344 & 274 & 2516 \\
\hline gm126 & - & - & 2135 & - & - & - & 1196 & 119 & 201 \\
\hline gm144 & - & - & - & - & 2806 & - & 2770 & 1461 & 1481 \\
\hline gm157 & - & - & 1914 & 2173 & - & - & 403 & 438 & 262 \\
\hline gm169 & - & - & - & - & 3146 & - & 2108 & 2971 & 962 \\
\hline gm179 & - & 1431 & 366 & 806 & 137 & - & 74 & 72 & 67 \\
\hline gm186 & - & - & - & - & 1674 & - & 951 & 842 & 223 \\
\hline gm187 & - & - & 2206 & 365 & 281 & - & 685 & 600 & 182 \\
\hline gm189 & - & - & - & - & - & - & - & - & 2961 \\
\hline $\operatorname{gm} 223$ & - & - & 2383 & - & 922 & - & 477 & 426 & 1473 \\
\hline $\operatorname{gm} 246$ & - & - & - & - & - & - & - & - & 1744 \\
\hline $\operatorname{gm} 256$ & - & - & - & - & - & - & - & - & 2291 \\
\hline $\operatorname{gm} 25$ & 1490 & - & 1387 & - & 653 & 2880 & 279 & 171 & 395 \\
\hline $\operatorname{gm} 269$ & - & - & - & 1656 & 452 & - & 1046 & 2948 & 1182 \\
\hline $\operatorname{gm} 275$ & - & - & - & - & - & - & 1180 & 2664 & 568 \\
\hline
\end{tabular}

strength of the consistencies. In almost all use cases, TRICs use less backtracks than EDAC, TRICs ${ }^{p}$ being the best. Compared to EDAC, (1) $\mathrm{TRICs}^{p}$ reduce the number of backtracks by $35 \%$ (2) $\mathrm{TRICs}^{r p}$ only slightly decrease the number of backtracks because of their reduced strength, and on many categories of benchmarks with a significantly reduced number of triangles they become almost equivalent to EDAC (3) TRICs ${ }^{r s}$ produce a smaller number of backtracks than TRICs ${ }^{r p}$ (thanks to the strengthened filtering during search) but still larger than $\mathrm{TRICs}^{p}$ (because of their significant reduced strength).

To summarize these experiments, it appears that some problems, as illustrated by the ChineseChars and GeomSurf7 cases, require the tightened lower 


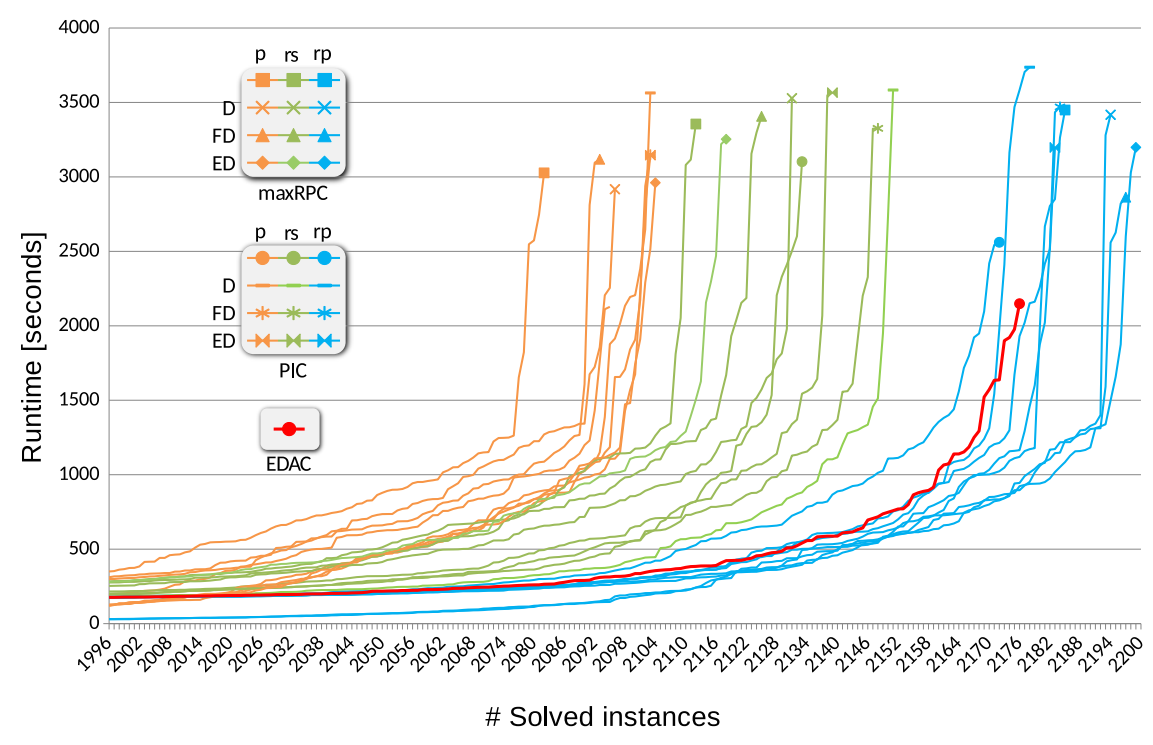

Fig. 17 Cactus plot on the full set of benchmarks. A point $(x, y)$ for a method $m$ on this diagram means that method $m$ is able to solve $x$ problems if a deadline of $y$ seconds is used for each problem independently.

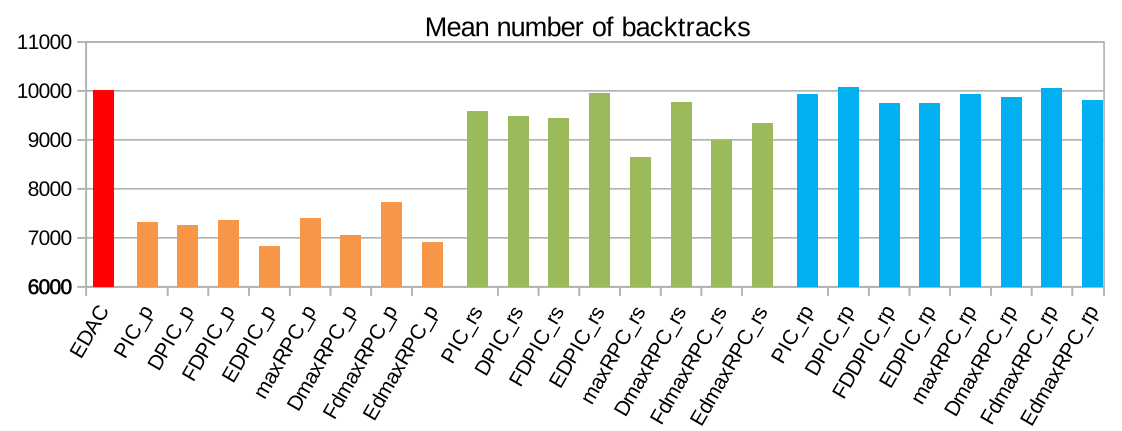

Fig. 18 The mean number of backtracks, computed on the overall set of benchmarks, that are used by EDAC and TRICs in the three use cases

bounds offered by TRICs to be solved. On these problems, when using TRICs for pre-processing, we can actually solve more instances in less time than EDAC. On these problems, restricted versions slightly reduce the advantage of TRICs. However, on problems having large triangle density, TRICs becomes significantly slower and thus solve less instances than EDAC. In these cases, using the restricted versions for pre-processing allows to improve the results. Finally, when the restricted TRICs are applied during both pre-processing and search, they always behave worse than when used for pre-processing only. 


\section{Conclusion}

In this paper, we have proposed six softening levels for strong triangle-based consistencies. This gives rise to eighteen soft extensions of hard RPC, PIC and maxRPC to CFNs. We have done a pairwise comparison of all these consistencies, among themselves and against their AC counterparts. We have shown that the new consistencies are strictly stronger than their AC counterparts in the sense that they provide tighter lower bounds than ACs. This improvement in lower bound is important for reducing the number of backtracks and for accelerating search. We have proposed algorithms for enforcing the soft consistencies of the PIC and maxRPC families. The experimentation shows that our soft consistencies are efficient when applied as a pre-processing on graphs with a relatively low triangle density such as ChineseChars and GeomSurf-7, defined on grid graphs. However, their performance decreases on graphs having a large triangle density. To make these soft consistencies practicable on problems where the number of triangles is large, we designed a restricted version by limiting the number of triangles to be processed. The best choice overall seems to be to use this restricted version for pre-processing and to switch to EDAC during search.

Acknowledgements This work has been partly funded by the "Agence nationale de la Recherche", reference ANR-10-BLA-0214.

\section{References}

1. Allouche, D., Bessiere, C., .Boizumault, P., Givry, S., Gutierrez, P., Loudni, S., Metivier, J., Schiex, T.: Decomposing global cost functions. In: Proc. of AAAI (2012)

2. Bensana, E., Lemaître, M., Verfaillie, G.: Earth observation satellite management. Constraints 4(3), 293-299 (1999)

3. Berlandier, P.: Improving domain filtering using restricted path consistency. In: Proceedings IEEE Conference on Artificial Intelligenece and Applications (CAIA'95) (1995)

4. Cabon, B., de Givry, S., Lobjois, L., Schiex, T., Warners, J.: Radio link frequency assignment. Constraints 4, 79-89 (1999)

5. Cooper, M., de Givry, S., Sanchez, M., Schiex, T., Zytnicki, M., Werner, T.: Soft arc consistency revisited. Artificial Intelligence 174, 449-478 (2010)

6. Cooper, M., de Givry, S., Sanchez, M., Schiex, T., Zytnicki, M.: Virtual Arc Consistency for Weighted CSP. In: Proc. of AAAI'2008. Chicago, USA (2008)

7. Cooper, M.C.: Reduction operations in fuzzy or valued constraint satisfaction. Fuzzy Sets and Systems 134(3), 311-342 (2003)

8. Cooper, M.C.: High-order consistency in Valued Constraint Satisfaction. Constraints 10, 283-305 (2005)

9. Cooper, M.C., de Givry, S., Schiex, T.: Optimal soft arc consistency. In: Proc. of IJCAI'2007, pp. 68-73. Hyderabad, India (2007)

10. Cooper, M.C., Schiex, T.: Arc consistency for soft constraints. Artificial Intelligence 154(1-2), 199-227 (2004)

11. Debruyne, R., Bessière, C.: From restricted path consistency to max-restricted path consistency. In: Proc. of CP'97, no. 1330 in LNCS, pp. 312-326. Springer-Verlag, Linz, Austria (1997)

12. Dehani, D., Lecoutre, C., Roussel, O.: Extension des cohérences wcsps aux tuples. In: Proc. of JFPC-13 (2013) 
13. Favier, A., de Givry, S., Legarra, A., Schiex, T.: Pairwise decomposition for combinatorial optimization in graphical models. In: Proc. of IJCAI'11. Barcelona, Spain (2011)

14. Freuder, E.C., Elfe, C.D.: Neighborhood inverse consistency preprocessing. In: Proc. of AAAI'96. Portland, OR (1996)

15. Hurley, B., O’Sullivan, B., Allouche, D., Katsirelos, G., Schiex, T., Zytnicki, M., de Givry, S.: Multi-Language Evaluation of Exact Solvers in Graphical Model Discrete Optimization. In: Proc. of CP-AI-OR'2016. Banff, Canada (2016)

16. Larrosa, J.: On arc and node consistency in weighted CSP. In: Proc. AAAI'02, pp. 48-53. Edmondton, (CA) (2002)

17. Larrosa, J., de Givry, S., Heras, F., Zytnicki, M.: Existential arc consistency: getting closer to full arc consistency in weighted CSPs. In: Proc. of the $19^{\text {th }}$ IJCAI, pp. $84-89$. Edinburgh, Scotland (2005)

18. Larrosa, J., Heras, F., de Givry, S.: A logical approach to efficient max-sat solving. Artif. Intell. 172(2-3), 204-233 (2008)

19. Larrosa, J., Schiex, T.: In the quest of the best form of local consistency for weighted CSP. In: Proc. of the $18^{\text {th }}$ IJCAI, pp. 239-244. Acapulco, Mexico (2003)

20. Larrosa, J., Schiex, T.: Solving weighted CSP by maintaining arc consistency. Artif. Intell. 159(1-2), 1-26 (2004)

21. Lee, J., Leung, K.: Towards efficient consistency enforcement for global constraints in weighted constraint satisfaction. In: Proc. of the $21^{r d}$ IJCAI, pp. 559-565. Pasadena (CA), USA (2009)

22. Lee, J., Leung, K.: Consistency techniques for flow-based projection-safe global cost functions in weighted constraint satisfaction. Artificial Intelligence 43, 257-292 (2012)

23. Sánchez, M., de Givry, S., Schiex, T.: Mendelian error detection in complex pedigrees using weighted constraint satisfaction techniques. Constraints 13(1-2), 130-154 (2008)

24. Schiex, T.: Arc consistency for soft constraints. In: Principles and Practice of Constraint Programming - CP 2000, LNCS, vol. 1894, pp. 411-424. Singapore (2000)

25. Schiex, T., Fargier, H., Verfaillie, G.: Valued constraint satisfaction problems: hard and easy problems. In: Proc. of the 14 ${ }^{\text {th }}$ IJCAI, pp. 631-637. Montréal, Canada (1995)

26. Simoncini, D., Allouche, D., de Givry, S., Delmas, C., Barbe, S., Schiex, T.: Guaranteed discrete energy optimization on large protein design problems. Journal of chemical theory and computation 11(12), 5980-5989 (2015)

27. Traoré, S., Allouche, D., André, I., de Givry, S., Katsirelos, G., Schiex, T., Barbe, S.: A new framework for computational protein design through cost function network optimization. Bioinformatics 29(17), 2129-2136 (2013) 